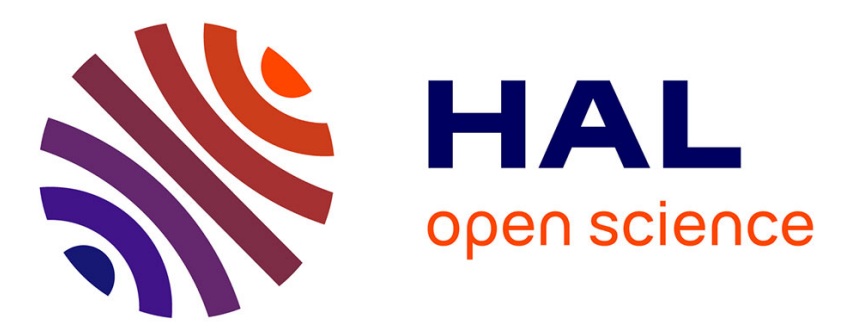

\title{
Specific absorption rate dependence on temperature in magnetic field hyperthermia measured by dynamic hysteresis losses (ac magnetometry).
}

Eneko Garaio, Olivier Sandre, Juan-Mari Collantes, Jose Angel Garcia, Stéphane Mornet, Fernando Plazaola

\section{To cite this version:}

Eneko Garaio, Olivier Sandre, Juan-Mari Collantes, Jose Angel Garcia, Stéphane Mornet, et al.. Specific absorption rate dependence on temperature in magnetic field hyperthermia measured by dynamic hysteresis losses (ac magnetometry).. Nanotechnology, 2015, 26 (1), pp.015704. 10.1088/09574484/26/1/015704 . hal-01226822

\section{HAL Id: hal-01226822 \\ https://hal.science/hal-01226822}

Submitted on 3 Nov 2018

HAL is a multi-disciplinary open access archive for the deposit and dissemination of scientific research documents, whether they are published or not. The documents may come from teaching and research institutions in France or abroad, or from public or private research centers.
L'archive ouverte pluridisciplinaire HAL, est destinée au dépôt et à la diffusion de documents scientifiques de niveau recherche, publiés ou non, émanant des établissements d'enseignement et de recherche français ou étrangers, des laboratoires publics ou privés. 


\title{
Specific Absorption Rate Dependence on Temperature in Magnetic Field Hyperthermia Measured by Dynamic Hysteresis Losses (AC Magnetometry)
}

\author{
Eneko Garaio*a $^{* a}$ Olivier Sandre ${ }^{\dagger \mathrm{b}}$, Juan-Mari Collantes ${ }^{\mathrm{a}}$, Jose Angel Garcia ${ }^{\mathrm{c}, \mathrm{d}}$, Stéphane Mornet ${ }^{\mathrm{e}}$, and \\ Fernando Plazaola ${ }^{\mathrm{a}}$ \\ ${ }^{a}$ Elektrizitatea eta Elektronika Saila, UPV/EHU, P.K. 644, Bilbao, Spain \\ ${ }^{\mathrm{b}}$ Laboratoire de Chimie des Polymères Organiques, UMR 5629 CNRS / Université de Bordeaux, Bordeaux, France \\ ${ }^{\mathrm{c}}$ Fisika Aplikatua II Saila, UPV/EHU, P.K. 644, Bilbao, Spain \\ ${ }^{\mathrm{d}}$ BC Materials (Basque Center for Materials, Application and Nanostructures), 48040 Leioa, Spain \\ ${ }^{\mathrm{e}}$ Institut de Chimie de la Matière Condensée de Bordeaux, UPR 9048 CNRS / Université de Bordeaux, Bordeaux, France
}

\begin{abstract}
Magnetic nanoparticles are intensively studied for their potential use for magnetic hyperthermia, a treatment that has passed a phase II clinical trial against severe brain cancer (glioblastoma) at the end of 2011. Their heating power, characterized by the "specific absorption rate $(S A R)$ ", is often considered temperature independent in the literature, mainly because of the difficulties that arise from the measurement methodology. Using a dynamic magnetometer presented in a recent paper, we measure here the thermal dependence of $S A R$ for superparamagnetic iron oxide (maghemite) nanoparticles (NPs) of four different size-ranges corresponding to mean diameters around $12 \mathrm{~nm}, 14 \mathrm{~nm}, 15 \mathrm{~nm}$ and $16 \mathrm{~nm}$. The article reports a parametrical study extending from 10 to $60{ }^{\circ} \mathrm{C}$ in temperature, from 75 to $1031 \mathrm{kHz}$ in frequency, and from 2 to $24 \mathrm{kA} \cdot \mathrm{m}^{-1}$ in magnetic field strength. It was observed that $S A R$ values of smaller NPs decrease with temperature whereas for the larger sample $(16 \mathrm{~nm}) S A R$ values increase with temperature. The measured variation of $S A R$ with temperature is frequency dependent. This behaviour is fully explained within the scope of linear response theory based on Néel and Brown relaxation processes, using independent magnetic measurements of the specific magnetization and the magnetic anisotropy constant. A good quantitative agreement between experimental values and theoretical values is confirmed in a tri-dimensional space that uses as coordinates the field strength, the frequency and the temperature.
\end{abstract}

*e-mail: eneko.garayo@ehu.es

† $e$-mail: olivier.sandre@enscbp.fr 


\section{Introduction}

The $S A R$ value, sometimes also denoted specific loss power (SLP), defined as the power transformed into heat per unit of mass of nanoparticles, drastically depends on external parameters such as the frequency and intensity of the applied magnetic field, as well as on the internal characteristics of the nanoparticles, i.e. size, shape, material, agglomeration state and even on properties (e.g. viscosity or thermal conductivity) of the dispersion medium [1, 2, 3, 4]. Moreover, a much less known fact is that $S A R$ can also depend on temperature for superparamagnetic nanoparticles (NPs). But although temperature explicitly appears in the linear approximated relaxation equations classically used to model magnetic field hyperthermia [2], experimental studies of the temperature-dependency of SAR are rather scarce.

Until now, $S A R$ values of magnetic nanoparticles were measured most often by calorimetric methods $[1,3,5,6]$. For a suspension of nanoparticles in a fluid carrier, the $S A R$ is obtained experimentally from the slope of the temperature curve by the formula [7]:

$$
S A R=\frac{m_{\mathrm{d}} \cdot C_{\mathrm{p}, \mathrm{d}}}{m_{\mathrm{NP}}} \cdot\left|\frac{d T}{d t}\right|_{t=0}
$$

where $m_{\mathrm{d}}$ and $C_{\mathrm{p}, \mathrm{d}}$ are the mass and heat capacity of the dispersion medium respectively, while $m_{\mathrm{NP}}$ is the mass of nanoparticles (including all the elements of the magnetic material, such as oxygen for oxides). However, the calorimetric methods present serious difficulties to measure the temperature dependency of given magnetic nanoparticle's heating power. Only when the sample holder is quasi-adiabatic and the surrounding temperature is properly controlled, it is possible to obtain the initial time slope in equation (1) at different temperatures. Natividad et al. measured by these means temperature-dependent SAR of commercial magnetite [8] and manganite perovskite [9] nanoparticles respectively in the 120 - 250 and 253 $343 \mathrm{~K}$ temperature ranges, i.e. around the blocking temperature $T_{\mathrm{B}}$ for iron oxide and near to the Curie temperature $T_{\mathrm{C}}$ for manganite. Regmi et al. [10] measured by calorimetry the temperature dependent magnetic dissipation of iron oxide nanoparticles suspended in frozen and liquid water at a single frequency of $395 \mathrm{kHz}$. They provided qualitative confirmation for the linear response theory based on Néel relaxation process.

There are several more convenient methods than the calorimetry one to measure $S A R$ values as a function of temperature. They are based on Faraday's law of induction to obtain $M(t)$, the dynamic magnetization $[11,12,13]$. The loss power values can be obtained directly from AC hysteresis loops area at different temperatures. Veverka et al. measured by this method the specific loss power of cobalt ferrite NPs in agarose gel at the single frequency of $50 \mathrm{kHz}$ and at $28 \mathrm{kA} \cdot \mathrm{m}^{-1}(35 \mathrm{mT})$ intensity [14]. The temperature range was from $25^{\circ} \mathrm{C}$ to $80^{\circ} \mathrm{C}$. Bekovic et al. obtained SAR values for maghemite NPs $10.9 \mathrm{~nm}$ in size dispersed in mineral oil in the temperature range of 20 to $80{ }^{\circ} \mathrm{C}$ at the single frequency of $100 \mathrm{kHz}$ [15]. 
In the present work, a previously tested lab-made AC magnetometer giving results compatible with the calorimetric method but with a higher sensitivity [16], is used to study the thermal dependence of $S A R$ values of different nanoparticle batches dispersed in water. The $S A R$ variations with temperature $\left(\triangle S A R_{\mathrm{T}}\right)$ of superparamagnetic maghemite nanoparticles of different size distributions have been measured in a wide applied magnetic field frequency range (from 75 to $1030 \mathrm{kHz}$ ). The observed thermal behaviour is clearly size dependent: for small samples, absorption rate decreases with temperature whereas for the largest sample, it increases. The change rate of $S A R$ with temperature is also frequency dependent, except for the smallest sized sample. The measured derivative of SAR versus temperature becomes more negative with frequency increase. We successfully explain the thermal behaviour of superparamagnetic samples considering Brown and Néel relaxation times. In that case we show a relationship between the thermal dependence of SAR (either decrease or increase) and the mean (number-average) diameter compared to the optimal value obtained by simulation of the conventional relaxation equations of magnetic moment [2].

\section{Experimental}

\subsection{Synthesis of Iron oxide NPs.}

Four samples made of superparamagnetic iron oxide were used in this study. Those denoted I, II, III were synthesized by alkaline co-precipitation of ferrous and ferric salts followed by different size sorting and coating steps, whereas sample IV was a gift of the Nanotex ${ }^{\circledR}$ company. Sample I has the broader size ranges, with the polydispersity of magnetic cores typical of the aqueous co-precipitation route. Then it was coated by citrate ligands in order to be stable in aqueous media around $\mathrm{pH} \sim 7$. For samples II and III, the maghemite nanoparticles right after the co-precipitation were dispersed in nitric acid. Then they were submitted to a size-sorting procedure based on the liquid-liquid phase-separation obtained by screening the electrostatic repulsions with an excess of electrolyte $\left(\mathrm{HNO}_{3}\right)$ concentration. The principle of this sorting is that the concentrated phase (denoted $\mathrm{C}$ ) is enriched with the larger nanoparticles, whereas the dilute phase (denoted S) contains the smaller nanoparticles. After repeating several phase separation and washing steps, samples II and III originate from fractions $\mathrm{C} 1 \mathrm{C} 2 \mathrm{C} 3$ and $\mathrm{C} 1 \mathrm{C} 2 \mathrm{~S} 3$, corresponding respectively to the pellet and the supernatant phases obtained by adding $\mathrm{HNO}_{3}$ electrolyte to the previous $\mathrm{C} 1 \mathrm{C} 2$ fraction and washing of the ions in excess. While sample II was kept in dilute $\mathrm{HNO}_{3}$ at $\mathrm{pH} \sim 2$, sample III was coated by Dextran in order to be dispersible in neutral aqueous media, in particular biological ones. For all samples, the iron oxide concentration was measured precisely by thermogravimetry analysis (TGA) and UV-visible spectroscopy, giving comparable results within few percents. 


\subsubsection{Broad size-distribution citrate-coated maghemite NPs (Sample I)}

Superparamagnetic iron oxide nanoparticles were synthesized by the Massart's synthesis of alkaline co-precipitation in water [17]. The resulting nanoparticles of magnetite $\left(\mathrm{Fe}_{3} \mathrm{O}_{4}\right)$ were oxidized into maghemite $\left(\gamma-\mathrm{Fe}_{2} \mathrm{O}_{3}\right)$ [18]. Then they were coated by tri-sodium citrate in order to obtain a stable colloidal suspension at $\mathrm{pH} \approx 7$ [19]. The hydrodynamic diameter measured by dynamic light scattering with a Malvern NanoZS90 instrument was $33 \mathrm{~nm}$, with a polydispersity index PDI $=0.30$ as determined by the 2 nd order cumulants fitting method of the auto-correlogram. The size-dispersity of these nanoparticles was assessed by automated particle-counting on TEM images (figure 1). Later the size distribution of the magnetic cores will also be obtained by another way, namely by fitting the static (DC) magnetization curve measured by a vibrating sample magnetometer (VSM), with the Langevin formalism of superparamagnetism. In both cases (TEM and VSM), a Log-normal distribution law was chosen to represent the inherent size-dispersity of the sample.

\subsubsection{Larger and narrower size-distribution maghemite NPs (Sample II)}

In order to obtain maghemite NPs with a higher heating efficiency, a size-grading method based on successive phase separation by added electrolyte was applied to isolate a fraction with a narrower size-distribution and a larger average diameter [20]. Contrary to Sample I, this refined fraction (Sample II) remained uncoated, i.e. dispersed in acidic medium $\left(\mathrm{HNO}_{3} \mathrm{pH} \sim\right.$ 2) thank to the cationic charges of their surface brought by adsorbed protons. The successive separation of the initial suspension by a series of addition of $\mathrm{HNO}_{3}$ to screen the electrostatic repulsions and washing steps to obtain fractions of refined size-grade is detailed in the supporting information of several references [21, 22]. The hydrodynamic diameter of Sample II diluted 400 times in $\mathrm{HNO}_{3}$ at $\mathrm{pH} \sim 2$ is $32 \mathrm{~nm}$ (from 5 successive measurements by dynamic light scattering at $25^{\circ} \mathrm{C}$ and $90^{\circ}$ scattering angle with a NanoZS90 instrument, Malvern, UK), with a low polydispersity index $\mathrm{PDI}=0.14$.

The weight concentration of iron oxide in sample II is $c_{0}{ }^{\mathrm{II}}=99 \mathrm{~g} \cdot \mathrm{L}^{-1}=9.9 \times 10^{4} \mathrm{~g} \cdot \mathrm{m}^{-3}$ as measured by UV-Vis spectroscopy (Fig. 2a). It was diluted by a factor $8\left(c_{\mathrm{II}}=12.3 \mathrm{~g} \cdot \mathrm{L}^{-1}\right)$ to perform dynamic hysteresis measurements at varying temperature. Proper $S A R$ measurements need an accurate determination of concentration of iron oxide in the suspensions. For that we used the comparison of the UV-Vis absorption spectrum from 240 to $800 \mathrm{~nm}$ to a calibration curve [23]. 


\subsubsection{Larger and narrower size-distribution maghemite NPs coated by Dextran (Sample III)}

The fraction $\mathrm{C} 1 \mathrm{C} 2 \mathrm{~S} 3$ that contains magnetic cores slightly smaller than C1C2C3 (Sample II) was coated by neutral hydrophilic polysaccharide chains of Dextran T70 (molar mass 70000 $\mathrm{g} \cdot \mathrm{mol}^{-1}$ ), that is a common method to disperse iron oxide nanoparticles in aqueous media at $\mathrm{pH} 7$, especially biological buffers $[24,25]$. After incubation with the polymer at $60{ }^{\circ} \mathrm{C}$, the nanoparticles were washed with ethanol to remove un-adsorbed polymer chains. The alcohol was eliminated by rotatory evaporation and the MNPs were readily re-dispersed in water. The concentration measured by TGA was $c_{0}{ }^{\mathrm{III}}=114 \mathrm{~g} \cdot \mathrm{L}^{-1}$. The hydrodynamic diameter of Sample III diluted 300 times in water is $56 \mathrm{~nm}$ with a PDI $=0.13$. It was diluted to $c_{\text {III }}=20.5 \mathrm{~g} \cdot \mathrm{L}^{-1}$ for the $S A R$ measurements.

\subsubsection{Commercial iron oxide NPs coated by poly(acrylate) chains (Sample IV)}

A commercial batch of water-based $\mathrm{Fe}_{3} \mathrm{O}_{4}$ nanoparticles was kindly provided by the Nanotex ${ }^{\circledR}$ Company (reference: NanoMag-32). However, this sample partially sedimented. Several attempts were made unsuccessfully to re-suspend the nanoparticles in water by hydrophilic polymer coatings. But due to their large magnetic core sizes, another strategy was adopted to impede the sedimentation. Instead of stabilization, gelation of the aqueous phase was obtained by adding the suspension into $2 \mathrm{wt}$. \% Agar (dissolved at $60{ }^{\circ} \mathrm{C}$ ), then letting it cool down to room temperature.

\subsection{SAR measurements by AC magnetometry}

The radiofrequency (RF) magnetic field was generated by an air coil connected in parallel with a resonant circuit fed by a linear power amplifier [26, 27]. Then, the dynamic magnetization of the sample, $M_{\mathrm{t}}$, and AC hysteresis loops were measured by a system of three pickup coils previously described in [16, 27]. Afterward, the specific absorption rate values were obtained from the following integral of the dynamic magnetization $v s$. applied field strength $H_{\mathrm{t}}$ :

$$
S A R=\frac{f}{c} \oint \vec{M}_{\mathrm{t}} \cdot d \vec{H}_{\mathrm{t}}
$$

where $f$ is the frequency and $c$ the weight concentration of iron oxide (not iron metal). The integration is done over the period of the oscillating magnetic field [16].

To measure the thermal dependence of $S A R$, the temperature of the sample was previously raised by a hot air gun heater or decreased by using a circulating water chiller. Then, $M_{\mathrm{t}}$ was measured continuously while sample was reaching room temperature $\left(25^{\circ} \mathrm{C}\right)$, each measure- 
ment of a whole hysteresis loop lasting around $5 \mathrm{sec}$ (thus in quasi isothermal conditions within $\pm 0.5^{\circ} \mathrm{C}$ ).. A fiber optic thermometer insensitive to RF magnetic fields (Opsens, Quebec, QC, Canada) with an outer diameter of $0.4 \mathrm{~mm}$ recorded temperature inside the vial filled with 1.5 $\mathrm{mL}$ of sample. 
(a)

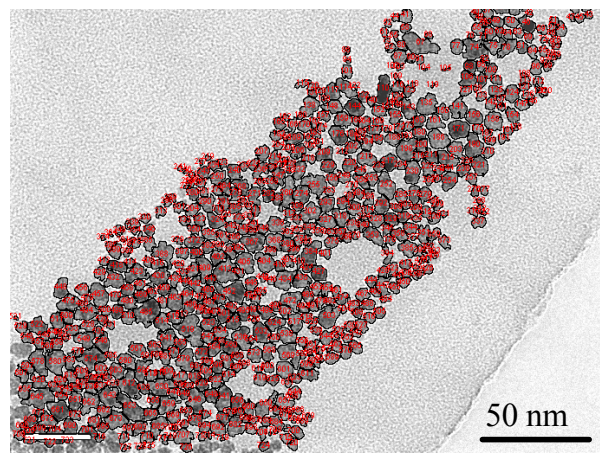

(c)

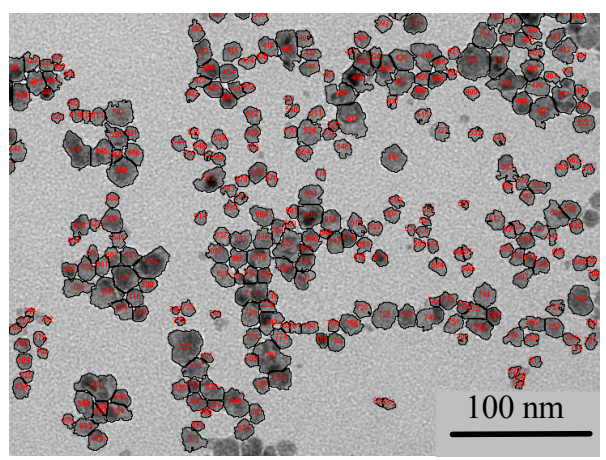

(b)

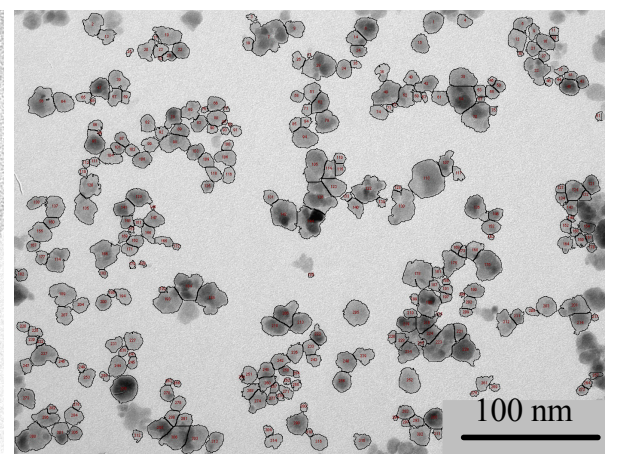

(d)

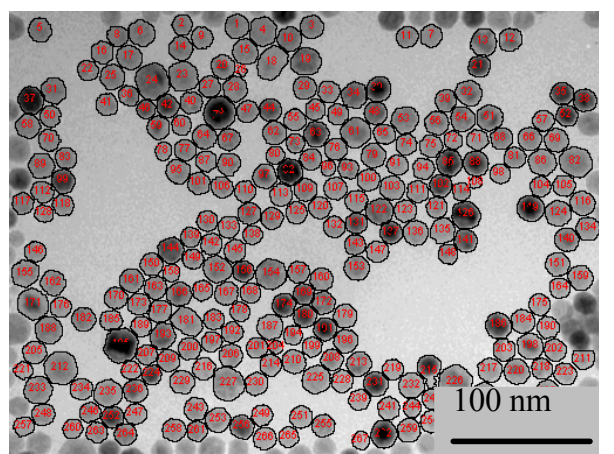

(e)

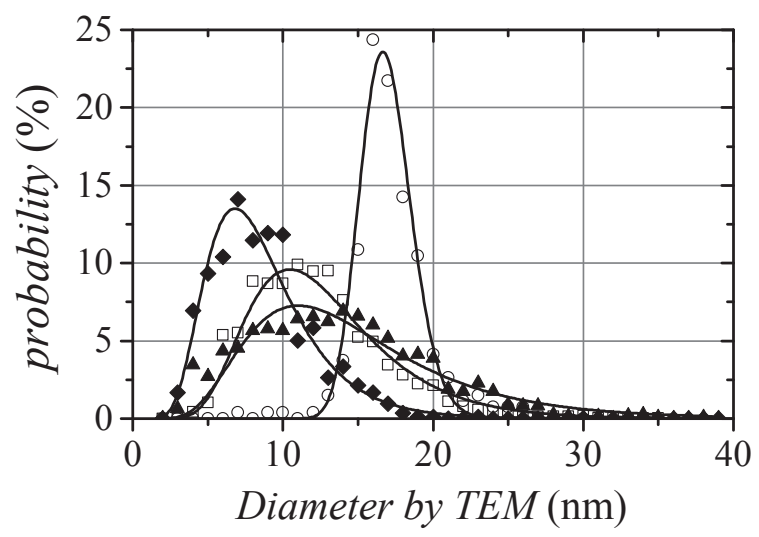

Figure 1: TEM images of the unsorted maghemite sample I (a), of the size-sorted fractions samples II $(b)$ and $I I I(c)$, and of the magnetite provided by Nanotex ${ }^{\circledR}$, sample $I V(d)$. The scale bars represent respectively $50 \mathrm{~nm}$ for sample I and $100 \mathrm{~nm}$ for the others. The images were processed with the ImageJ software (http://rsb.info.nih.gov/ij/) with background subtract, threshold, binary and watershed filters to detect the border lines of the nanoparticles and enable an automatic counting; e) Histograms of diameters for sample I (filled diamonds), sample II (filled triangles), sample III (empty squares) and sample IV (open circles). The statistics was built by processing 2 TEM images of sample I ( $N \sim 840$ nanoparticles), 5 images of sample II $(N \sim 1600)$, 3 images of sample III (N 2060), and 1 image of sample IV (N 270). The dotted and continuous lines are Log-normal fits of parameters $\alpha$ (median) and $\beta$ (standard width of the logarithms). Then equations (9) and (10) were used to compute the number-averages and standard deviations of diameters: $d_{T E M}=8.7 \pm 3.3 \mathrm{~nm}$ for sample I, $d_{T E M}=14.9 \pm 6.4 \mathrm{~nm}$ for sample II, $d_{T E M}=12.9 \pm 4.6 \mathrm{~nm}$ for sample III, and $d_{T E M}=16.9 \pm 1.7 \mathrm{~nm}$ for sample $\mathrm{IV}$; 
(a)

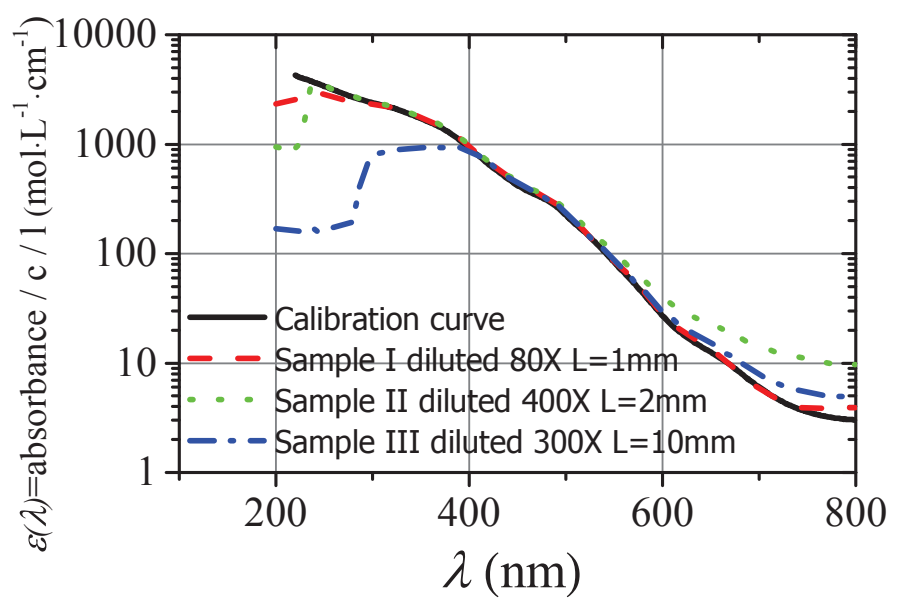

(b)

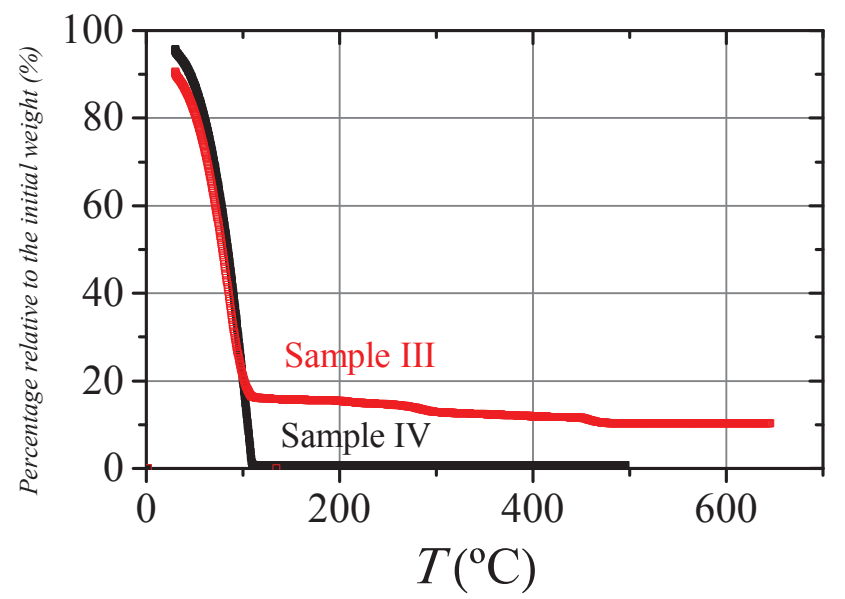

Figure 2: Non-destructive determination of the iron oxide concentration by UV-Vis spectroscopy. The spectra of the samples diluted respectively 80 times in water (sample I) and 400 times in $\mathrm{HNO}_{3}$ at $\mathrm{pH} 2$ (sample II) were recorded between 240 and $800 \mathrm{~nm}$. The extinction coefficient $\varepsilon(\lambda)$ calculated after normalization by the light path $L(\mathrm{~cm})$ and the equivalent iron concentration (respectively $[\mathrm{Fe}]_{I}=1.0 \mathrm{~mol} \cdot \mathrm{L}^{-1},[\mathrm{Fe}]_{I I}=$ $1.24 \mathrm{~mol} \cdot \mathrm{L}^{-1}$, and $[\mathrm{Fe}]_{I I I}=1.26 \mathrm{~mol} \cdot \mathrm{L}^{-1}$ as determined here) was fitted to a calibration curve obtained from measurements on samples at concentrations titrated by atomic emission spectroscopy. Then the weight concentration was simply deduced by multiplying by $80 \mathrm{~mol} \cdot \mathrm{L}^{-1}$ ( $\sim$ half the molar mass of $\gamma-\mathrm{Fe}_{2} \mathrm{O}_{3}$ ), yielding $c_{0}^{I}=80 \mathrm{~g} \cdot \mathrm{L}^{-1}$ for sample I, $c_{0}^{I I}=99 \mathrm{~g} \cdot \mathrm{L}^{-1}$ for sample II, and $c_{0}^{I I I}=100.6 \mathrm{~g} \cdot \mathrm{L}^{-1}$ for sample III. This method was not possible for sample IV because of its poor colloidal stability (inducing a strong scattering baseline to the spectrum); b) Thermogravimetry analysis (TGA) of samples III and IV leading to the inorganic dry weight concentrations, $c_{0}^{I I I}=114.3 \mathrm{~g} \cdot \mathrm{L}^{-1}$ for sample III and $c_{0}^{I V}=7.1 \mathrm{~g} \cdot \mathrm{L}^{-1}$ for sample IV. For sample III, the inflection point near $280^{\circ} \mathrm{C}$ corresponds to the degradation of Dextran under $\mathrm{N}_{2}$ atmosphere, while the second inflection point at $450^{\circ} \mathrm{C}$ is ascribed to the introduction of $\mathrm{O}_{2}$ and the burning of all the remaining organic coating. The weight ratio of polymer to iron oxide is $52 \%$. In the case of sample IV, no degradation of polymer coating is observed, which explains the weak colloidal stability of this sample (supposedly stabilized by poly (acrylic acid), according to Nanotex ${ }^{\circledR}$ ). 


\section{Results and discussion}

Hysteresis-loops for $\gamma-\mathrm{Fe}_{2} \mathrm{O}_{3}$ samples were measured at magnetic field intensities below 20 $\mathrm{kA} \cdot \mathrm{m}^{-1}$, frequencies of $75,532,1030 \mathrm{kHz}$, and at different sample temperatures ranging from 10 to $60^{\circ} \mathrm{C}$. Figure 3 shows the measured hysteresis cycles at different frequencies for samples I, II, III and IV. The DC magnetization curves at room temperature plotted on figure 3 have been obtained using a vibrating sample magnetometer (VSM). As expected, for all samples, the hysteresis area broadens from a single line at DC to a wide hysteresis loop as the frequency increases. This broadening is less pronounced in sample IV. In table 1, the colloidal properties of each sample are written. The corresponding volume fractions are given by $\phi=c / \rho$ where the mass density of maghemite is $\rho=5000 \mathrm{~kg} \cdot \mathrm{m}^{-3}$.

Table 1: Colloidal properties of the measured samples. For sample IV in agar-gel $d_{H}$ is assumed to be infinity.

\begin{tabular}{cccc}
\hline Sample & $\begin{array}{c}\text { Concentration } \\
c\left(\mathrm{mg} \cdot \mathrm{mL}^{-1}\right)\end{array}$ & $\begin{array}{c}\text { Volume fraction } \\
\phi(\%)\end{array}$ & $\begin{array}{c}\text { Hydrodynamic } \\
\text { diameter } d_{\mathrm{H}}(\mathrm{nm})\end{array}$ \\
\hline I & 8 & 0.16 & 33 \\
II & 12.3 & 0.245 & 32 \\
III & 20 & 0.4 & 56 \\
IV & 2 & 0.04 & $\infty$ \\
\hline
\end{tabular}

For each frequency, field intensity and temperature, the $S A R$ values were obtained by equation (2). Figure 2 shows the measured $S A R$ values for the maghemite samples, within the temperature range from 10 to $60{ }^{\circ} \mathrm{C}$. The different applied magnetic field frequencies are 75 , 532 and $1030 \mathrm{kHz}$ whereas the field intensity is $20 \mathrm{kA} \cdot \mathrm{m}^{-1}$ for samples I and II, $15 \mathrm{kA} \cdot \mathrm{m}^{-1}$ for sample III and $5 \mathrm{kA} \cdot \mathrm{m}^{-1}$ for sample IV. An almost linear drop of the specific heating power with temperature is observed in the case of samples I, II and III. Similar behaviour has been found by Bekovic et al. [15] who reported a decrease of $S A R$ values by $50 \%$ from 10 to $60{ }^{\circ} \mathrm{C}$ for $11 \mathrm{~nm}$ size maghemite nanoparticles measured by AC magnetometry at applied magnetic field frequency of $100 \mathrm{kHz}$. For sample IV, however, an increment of $S A R$ with temperature is observed at 532 and $1030 \mathrm{kHz}$ frequencies.

Figure 5 and 6 show the relative change of SAR with temperature (in \%) called here $\triangle S A R_{\mathrm{T}}$ and calculated by equation (3). As it can be appreciated, each sample presents a different thermal behaviour of its absorption rate: In sample IV $\triangle S A R_{\mathrm{T}}$ is positive at 532 and $1030 \mathrm{kHz}$ frequencies whereas it is negative at $75 \mathrm{kHz}$. In samples II and III $\triangle S A R_{\mathrm{T}}$ also depends on frequency but it is negative at all frequencies. $\triangle S A R_{\mathrm{T}}$ is negative too in sample I although in this case, it is frequency independent.

$$
\Delta S A R_{\mathrm{T}}=\frac{S A R(T)-S A R\left(T=10^{\circ} C\right)}{S A R\left(T=10^{\circ} C\right)}
$$


(a) Sample I

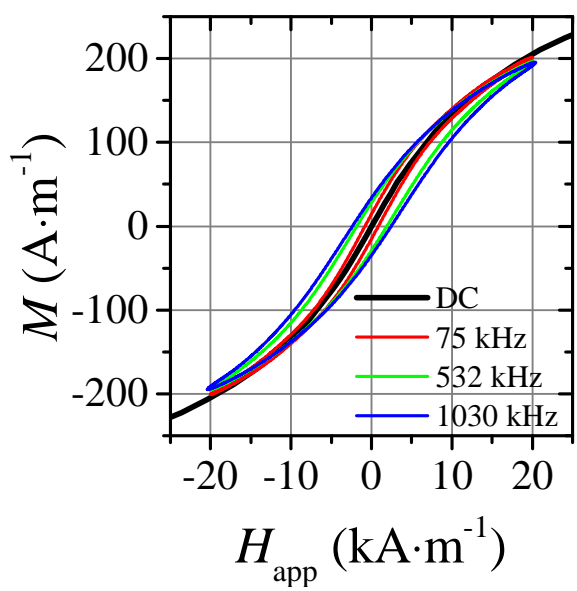

(c) Sample III

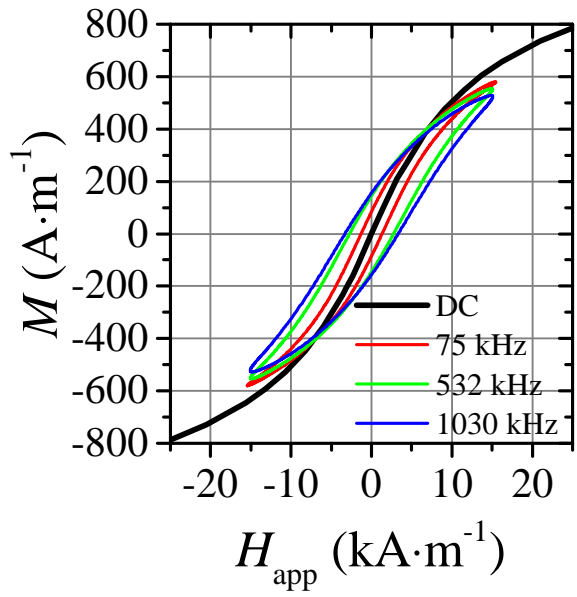

(b) Sample II

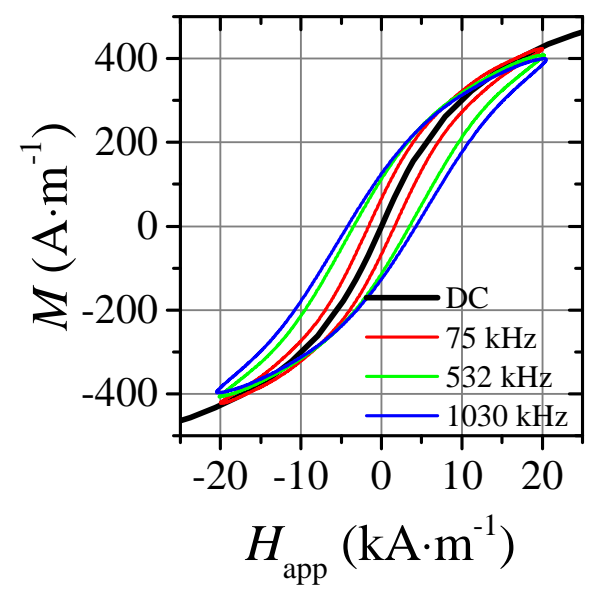

(d) Sample IV

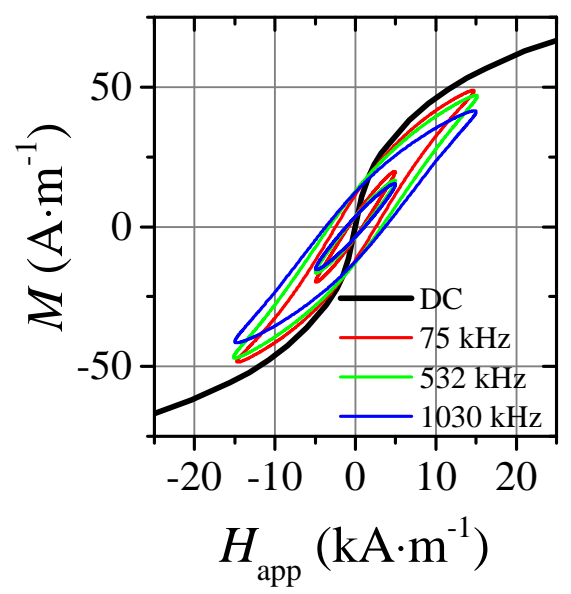

Figure 3: Hysteresis-loops measured at different frequencies for sample I, II , III and IV. DC magnetization curves (black line) were measured by VSM. For sample IV, the minor loops at $5 \mathrm{kA} \cdot \mathrm{m}^{-1}$ are plotted. Note that as the frequency increases, the loops broaden, except for sample IV, for which the area remains quasi-constant, leading to a SAR proportional to frequency (see figure 10d). The loops were measured at room temperature $\left(25^{\circ} \mathrm{C}\right)$.

The magnitude $\triangle S A R_{\mathrm{T}}$ has shown to be independent of applied magnetic field intensity for samples I, II and III. However, $\triangle S A R_{\mathrm{T}}$ is quite dependent on intensity in the case of sample IV. Figure 6 shows the measured $\triangle S A R_{\mathrm{T}}$ values for sample IV at low $\left(5 \mathrm{kA} \cdot \mathrm{m}^{-1}\right)$ and high $(15$ $\mathrm{kA} \cdot \mathrm{m}^{-1}$ ) field intensities. As it can be appreciated, at $75 \mathrm{kHz} S A R$ decreases more rapidly with temperature. At higher frequency $(532$ and $1030 \mathrm{kHz}), \Delta S A R_{\mathrm{T}}$ becomes negative when a larger magnetic field is applied. 
(a) Sample I

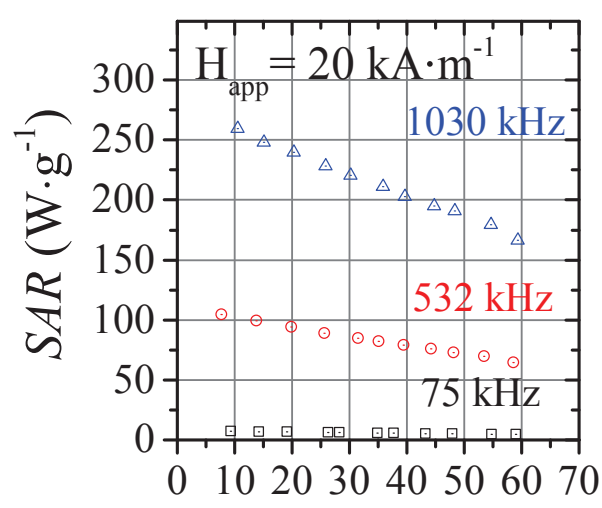

$T\left({ }^{\circ} \mathrm{C}\right)$ (b) Sample II

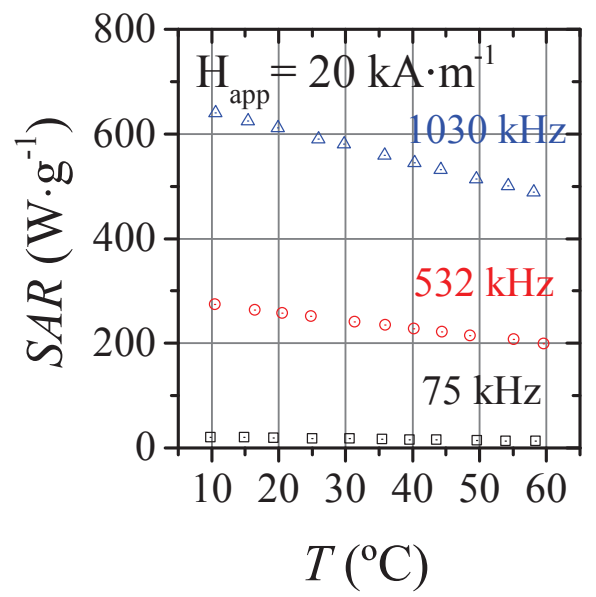

(d) Sample IV

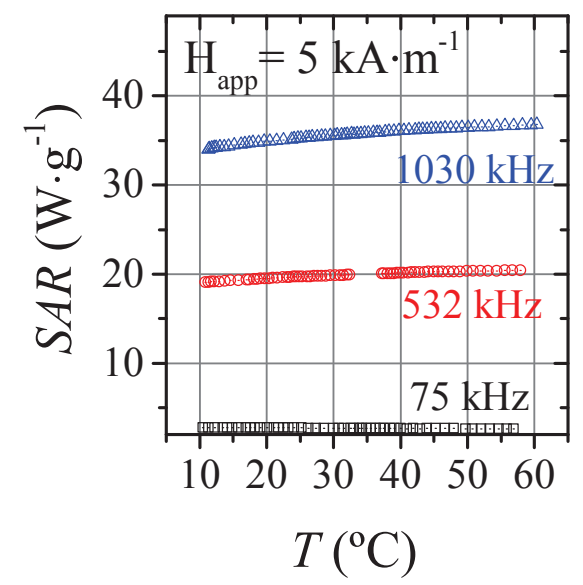

Figure 4: Measured SAR values dependence with temperature. The magnetic field intensity is $20 \mathrm{kA} \cdot \mathrm{m}^{-1}$ for samples I and II, $15 \mathrm{kA} \cdot \mathrm{m}^{-1}$ for sample III and $5 \mathrm{kA} \cdot \mathrm{m}^{-1}$ for sample IV. The magnetic field frequencies are 75 , 531 and $1031 \mathrm{kHz}$. 
(a) Sample I

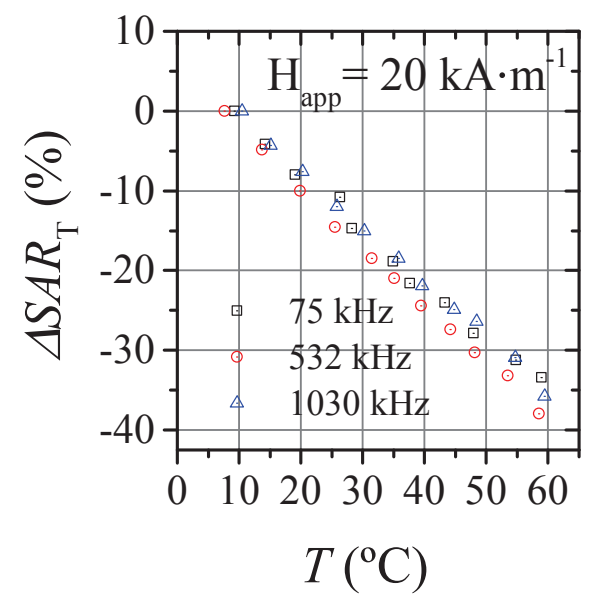

(b) Sample II

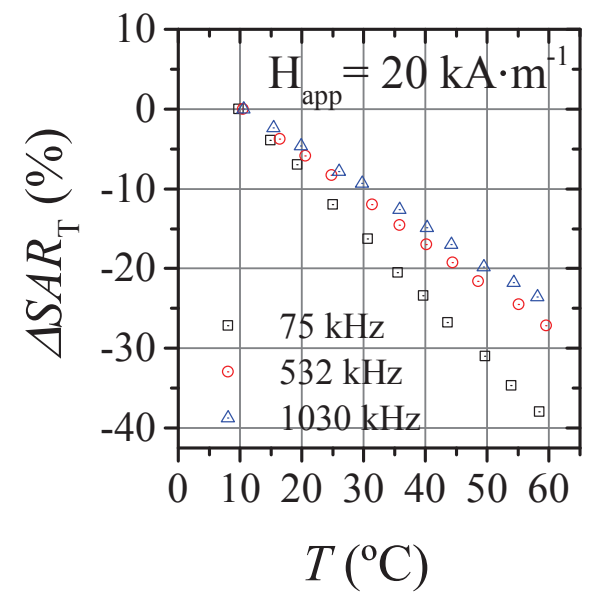

(c) Sample III

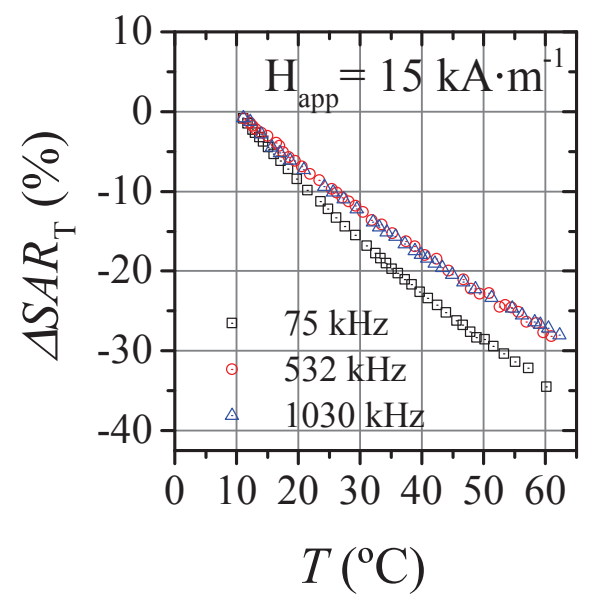

Figure 5: Measured $\triangle S A R_{T}$ values (see equation 3). The magnetic field intensity, $H_{\text {app }}$, is $20 \mathrm{kA} \cdot \mathrm{m}^{-1}$ for samples I and II whereas it is $15 \mathrm{kA} \cdot \mathrm{m}^{-1}$ for sample III. The magnetic field frequencies are 75, 531 and $1031 \mathrm{kHz}$ (square, circle and triangle symbols respectively).

(a)

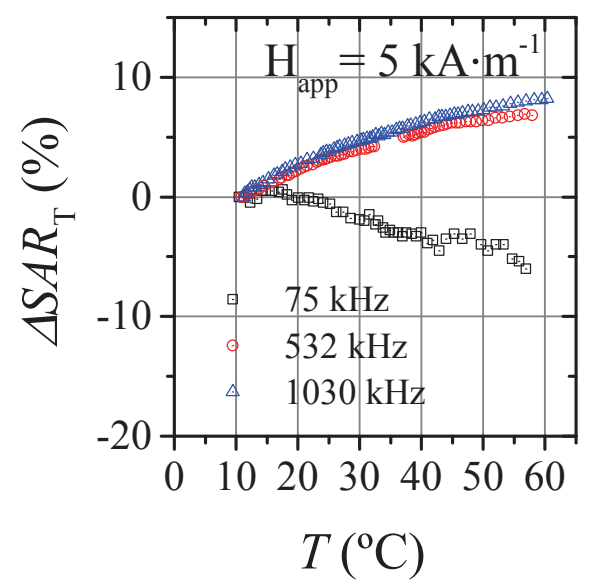

(b)

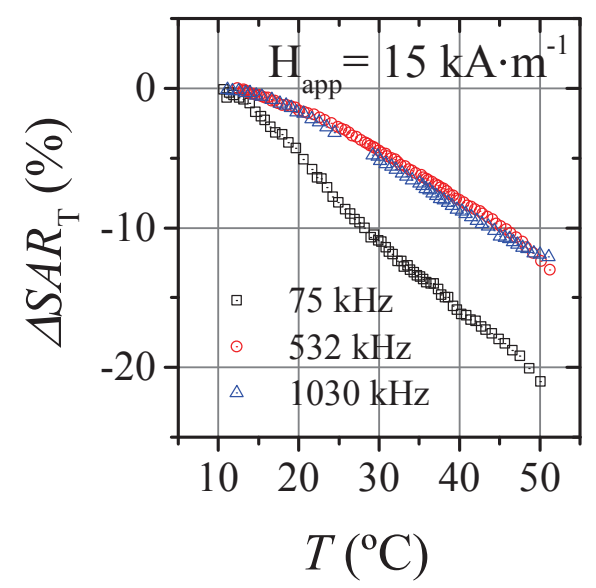

Figure 6: Measured $\triangle S A R_{T}$ values for sample IV at $5 \mathrm{kA} \cdot \mathrm{m}^{-1}$ (left) and $15 \mathrm{kA} \cdot \mathrm{m}^{-1}$ (right). 


\subsection{Linear response theory}

The linear-response theory is used to fit the measured experimental data and to explain the behaviour of $S A R$ with frequency, field strength and temperature observed in superparamagnetic $\gamma-\mathrm{Fe}_{2} \mathrm{O}_{3}$ nanoparticles. To do so, the effective relaxation time, $\tau_{\text {eff }}$, is computed by the combination of Néel relaxation time, $\tau_{\mathrm{N}}$, and Brownian time constant, $\tau_{\mathrm{B}}$, that is smaller than the shorter time between the two [2, 28]:

$$
\begin{gathered}
\tau_{\mathrm{B}}=\frac{3 \eta V_{\mathrm{H}}}{k_{\mathrm{B}} T} \\
\tau_{\mathrm{N}}=\tau_{0, \mathrm{~N}} \cdot \exp \left(\frac{K V_{\mathrm{M}}}{k_{\mathrm{B}} T}\right) \\
\tau_{e f f}=\frac{\tau_{\mathrm{N}} \cdot \tau_{\mathrm{B}}}{\tau_{\mathrm{N}}+\tau_{\mathrm{B}}}
\end{gathered}
$$

where $K$ is the anisotropy constant (in $\mathrm{J} \cdot \mathrm{m}^{-3}$ ), $V_{\mathrm{M}}=\pi \cdot d^{3} / 6$ the magnetic core volume, $V_{\mathrm{H}}=\pi \cdot d_{H}^{3} / 6$ the hydrodynamic volume, $T$ the temperature, $k_{\mathrm{B}}$ the Boltzmann constant and $\eta$ the viscosity coefficient of water (in Pa.s). In (5), $\tau_{0, \mathrm{~N}}$ is a characteristic attempt time with magnitude order of $10^{-13}-10^{-8}$ s [29,30]. A more complicated equation for the Néel time was introduced by R. Rosenweig [2], but we checked that it leads to similar results for the fitting of the experimental $S A R$ data. Afterwards, the theoretical $S A R$ values (in $\mathrm{W} \cdot \mathrm{g}^{-1}$ ) are obtained by the following expression [2]:

$$
S A R=\frac{1}{\rho} \mu_{0} \pi f H_{\mathrm{app}}^{2} \int_{0}^{\infty} d r \cdot g_{\mathrm{d}_{0}, \sigma}(2 r) \cdot \chi_{0} \cdot \frac{2 \pi \cdot f \cdot \tau_{\mathrm{eff}}}{1+\left(2 \pi \cdot f \cdot \tau_{\mathrm{eff}}\right)^{2}}
$$

where $\mu_{0}$ is the magnetic permeability of vacuum, $f$ is the frequency, $H_{\text {app }}$ the intensity of applied magnetic field, $\rho$ the nanoparticle mass density (in $\mathrm{g} \cdot \mathrm{m}^{-3}$ ) and $\tau_{\text {eff }}$ the relaxation time given by equation (6). The integral of equation (7) is performed for all nanoparticle size distribution $g_{\mathrm{d}_{0}, \sigma}(d)$, which is assumed to have a log-normal distribution:

$$
g_{\mathrm{d}_{0}, \sigma}(d)=\frac{1}{d \cdot \beta \sqrt{2 \pi}} \cdot \exp \left(\frac{-\ln (d / \alpha)^{2}}{2 \beta^{2}}\right)
$$

with $\alpha$ the median value of the distribution and $\beta$ the standard width of the Log values. Then the mean diameter (number-average) $d_{0}$ is given by:

$$
d_{0}=\alpha \cdot \exp \left(\beta^{2} / 2\right)
$$

and the standard deviation $\sigma$ is computed by: 


$$
\sigma=d_{0} \sqrt{\exp \left(\beta^{2}\right)-1}
$$

The parameter $\chi_{0}$ on equation (7) is the equilibrium susceptibility of these superparamagnetic samples. As an approximation [2], it can be considered as the static volume susceptibility per nanoparticle given by the Langevin function $L(x)$ :

$$
\chi_{0}=\frac{M_{\mathrm{d}}}{H_{\mathrm{app}}} \cdot L\left(\mu_{0} \frac{M_{\mathrm{d}} V_{\mathrm{M}}}{k_{\mathrm{B}} T}\right)
$$

where $M_{\mathrm{d}}$ is the magnetic domain magnetization of the nanoparticles (in $\mathrm{A} \cdot \mathrm{m}^{-1}$ ). Note that the parameter $\chi_{0}$ is size dependent and thus, it is placed into the integral of equation (7). The domain magnetization $M_{\mathrm{d}}$ in equation (11) is related to the saturation magnetization of the suspension, $M_{\mathrm{sat}}$, and the volume fraction of the suspension, $\phi$, by:

$$
M_{\mathrm{sat}}=\phi \cdot M_{\mathrm{d}}
$$

In order to calculate $S A R$ by means of equation (7), adequate values have to be used to estimate the two characteristic times. For the Brown's relaxation mechanism of the particles, temperature-dependent water viscosity $\eta$ listed in table 2 and hydrodynamic volume $V_{\mathrm{H}}$ computed from the hydrodynamic diameter $\left(d_{\mathrm{H}}\right)$ measured by DLS (see table 1) were used. In the case of sample IV, the nanoparticles exhibit much less rotational mobility because the agarose gel impedes particle rotation and hence, Brown time constant $\tau_{\mathrm{B}}$ can be considered infinite and the effective relaxation time $\tau_{\text {eff }}$ equals to Néel relaxation time $\tau_{\mathrm{N}}$ (see eq. 6). Also, the adequate values of $\log$ normal distribution parameters $\left(d_{0}\right.$ and $\sigma$ ), mono-domain magnetization $M_{\mathrm{d}}$ and crystal anisotropy $K$ have to be used to estimate Néel relaxation time for each sample. Moreover, in order to calculate the absorbed power at different temperatures, the thermal behaviour of magnetic properties must be known. In order to obtain these values and their thermal evolution, we have realized the following measurements: room temperature DC magnetization, field-cooled (FC) and zero-field cooled (ZFC) magnetization as a function of temperature. More precisely, temperature was initially decreased down to $\mathrm{T}=5 \mathrm{~K}$ under zero field (ZFC) or under $H=795.8 \mathrm{~A} \cdot \mathrm{m}^{-1}(10 \mathrm{Oe})(\mathrm{FC})$ and then magnetization was recorded while raising back to room temperature under a weak magnetic field $H=795.8 \mathrm{~A} \cdot \mathrm{m}^{-1}$ (10 Oe). 
Table 2: Water viscosity coefficient at different temperatures [31]

\begin{tabular}{cc}
\hline$T\left({ }^{\circ} \mathrm{C}\right)$ & $\eta\left(\mathrm{mPa} \cdot \mathrm{s}^{-1}\right)$ \\
\hline 10 & 1.307 \\
20 & 1.002 \\
30 & 0.797 \\
40 & 0.653 \\
50 & 0.547 \\
60 & 0.467 \\
\hline
\end{tabular}

\subsection{Room temperature DC magnetization curves}

Figure 5 shows DC magnetization curves measured at room temperature by vibrating sample magnetometer (VSM) for samples I and II dispersed in water with nanoparticle concentrations written on table 1 (same as for the SAR measurements). The absence of coercive field indicates that they are in the superparamagnetic (SPM) regime and hence, the magnetization, $M_{\mathrm{SPM}}$ can be described by the Langevin function (see equation 13) integrated for all sizes [32, 33]:

$$
M_{\mathrm{SPM}}=M_{\mathrm{sat}} \int_{0}^{\infty} d r \cdot g_{\mathrm{d}_{0}, \sigma}(2 r) \cdot L\left(\mu_{0} \frac{M_{\mathrm{d}} V_{\mathrm{M}}}{k_{\mathrm{B}} T} H_{\mathrm{app}}\right)
$$

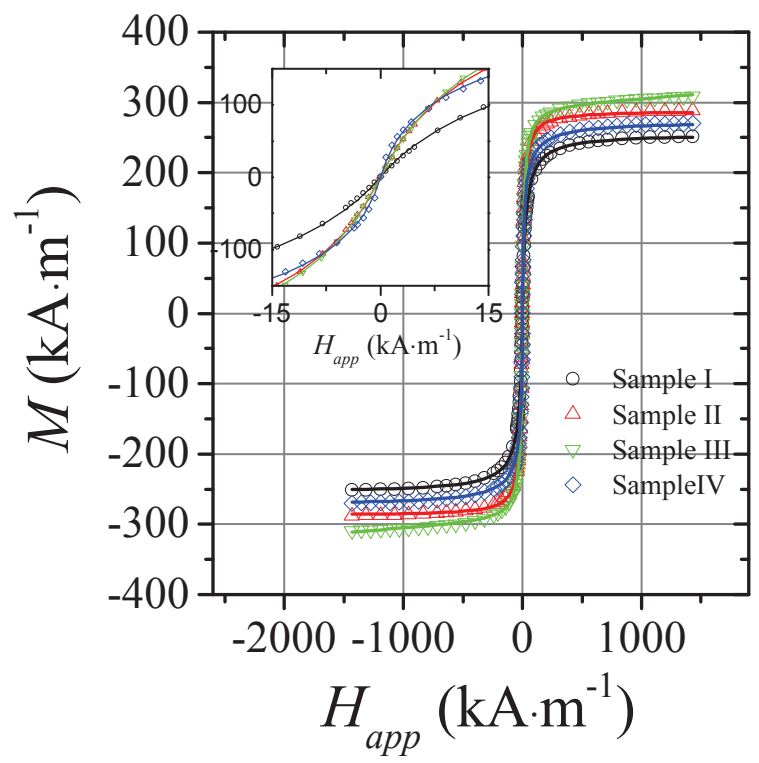

Figure 7: DC magnetization curves measured by VSM. Solid lines indicate the fitting of experimental data to equation (13).

Fitting the experimental data to equation (13), the values of mean nanoparticle sizes $d_{0}$ VSM and the standard deviations $\sigma^{\text {VSM }}$ can be obtained (note the superscript VSM). From the saturation magnetization $M_{\mathrm{sat}}$ and the measured volume fractions, the domain magnetization $M_{\mathrm{d}}$ 
can be obtained by means of equation (12). Table 3 shows the so obtained values at room temperature for samples I, II, III and IV. These values are compatible, within the experimental uncertainty, with the mean diameters and standard deviations measured by TEM: $d_{0}{ }^{\mathrm{TEM}}=8.7$ $\mathrm{nm}$ with $\sigma^{\mathrm{TEM}}=3.3 \mathrm{~nm}$ for sample $\mathrm{I}, d_{0}{ }^{\mathrm{TEM}}=14.9 \mathrm{~nm}$ with $\sigma^{\mathrm{TEM}}=6.4 \mathrm{~nm}$ for sample II, $d_{0}{ }^{\text {TEM }}=12.9 \mathrm{~nm}$ with $\sigma^{\text {TEM }}=4.6 \mathrm{~nm}$ for sample III, and $d_{0}{ }^{\text {TEM }}=16.9 \mathrm{~nm}$ with $\sigma^{\text {TEM }}=1.7 \mathrm{~nm}$ for sample IV. Diameters of SPM nanoparticles measured by TEM are indeed always slightly larger than the diameters deduced by magnetometry, as ascribed to a usual nonmagnetic dead layer around ferrite cores [34]. The tiny differences between diameters deduced from VSM and TEM measurements may arise from the fact that in microscopy, despite the high number $N$ of particles analysed by automated particle counting (from 270 to 2060), only a fraction of nanoparticles in the sample contributes to the final values of the diameter, whereas in VSM, the contribution of all the nanoparticles in the sample are averaged. The standard deviation $\sigma^{\mathrm{VSM}}$ is too large in the case of sample IV, which might be the sign of the presence of ferromagnetic nanoparticles in the suspension. In fact, it will be shown that the standard deviation $\sigma$ that best fit experimental $S A R$ values is always bellow $\sigma^{\mathrm{VSM}}$.

Table 3: Parameters that best fit the measured DC magnetization curves in figure 7 using equation (13). Next columns: Average sizes, standard size deviation and polydispersity index (p.i. ${ }^{\text {TEM }}$ ) obtained by TEM. For both techniques, the p.i. is equal to the beta parameter in the Log-normal distribution law (eq. 8).

\begin{tabular}{|c|c|c|c|c|c|c|c|}
\hline & $\begin{array}{c}M_{\mathrm{d}} \\
\left(\mathrm{kA} \cdot \mathrm{m}^{-1}\right)\end{array}$ & $\begin{array}{c}d_{0} \text { VSM } \\
(\mathrm{nm})\end{array}$ & $\begin{array}{c}\sigma^{\mathrm{VSM}} \\
(\mathrm{nm})\end{array}$ & p.i. VSM & $\begin{array}{c}d_{0}{ }_{(\mathrm{nm})}^{\mathrm{TEM}} \\
\text {. }\end{array}$ & $\begin{array}{c}\sigma^{\mathrm{TEM}} \\
(\mathrm{nm})\end{array}$ & $p . i .^{\mathrm{TEM}}$ \\
\hline Sample I & 254 & 12.9 & 4.9 & 0.38 & 8.7 & 3.3 & 0.38 \\
\hline Sample II & 283 & 14.9 & 4.4 & 0.29 & 14.9 & 6.4 & 0.41 \\
\hline Sample III & 295 & 14.5 & 4.3 & 0.29 & 12.9 & 4.6 & 0.30 \\
\hline Sample IV & 276 & 16.1 & 9.0 & 0.52 & 16.9 & 1.7 & 0.10 \\
\hline
\end{tabular}

\subsection{Zero-field cooled (ZFC) magnetization as a function of temperature}

The magnetization versus temperature curve of a sample of nanoparticles at small applied field is a source of valuable information about its magnetic properties. The experimental data can be described by a simple non-interacting model and hence, parameters like the anisotropy constant, between others, can be obtained. Here, in order to obtain the effective anisotropy constants $\left(K_{\text {eff }}\right)$, the ZFC-FC magnetization curves of the nanoparticle samples were measured. Afterward, the ZFC branches were fitted to a non-interacting moments linear model. This procedure was used by Denardin et al. [35] for the evaluation of effective anisotropy constant ( $\left.K_{\text {eff }}\right)$ in $\mathrm{Co}_{\mathrm{x}}\left(\mathrm{SiO}_{2}\right)_{1-\mathrm{x}}$ particles and by Nadeem et al. [36]. Because the model involves non-interacting moments, small concentrations of nanoparticles are preferred and hence, the samples were diluted 10 times when measuring ZFC magnetization curves. Due to the low magnetization of the 
diluted samples, a rf-SQUID based [37] commercial magnetometer (Quantum Designs MPMS7T) was used. A resolution of $10^{-7} \mathrm{emu}$ is commonly achieved with this type of commercial magnetometers [38]. In particular, the sensitivity of the MPMS-7T magnetometer, as specified by the manufacturers, is $6 \times 10^{-7} \mathrm{emu}$; enough to measure the magnetization of diluted samples, which was always larger than $5 \times 10^{-4} \mathrm{emu}$. This magnetometer was previously calibrated by Yttrium Iron Garnet $1 \mathrm{~mm}$ in diameter Sphere (Standard Reference Materials 2853) and reset after each measurement.

The magnetization of polydisperse non-interacting superparamagnetic nanoparticles, $M_{\mathrm{SPM}}$ is given by equation (13) in the previous section. For small applied magnetic field $H_{\text {app }}$ typical of FC/ZFC experiments, the Langevin function can be simplified to $L(x) \approx x / 3$. Above a critical nanoparticle volume, $V_{\mathrm{B}}$, the thermal energy is not enough to spontaneously reverse the magnetic moment and hence nanoparticles are no longer superparamagnetic (their magnetic moments are blocked). The value of $V_{\mathrm{B}}$ is given by the following well known phenomenological equation [39]:

$$
V_{\mathrm{B}}=\frac{k_{\mathrm{B}} T}{K} \cdot \ln \left(\frac{\tau_{\mathrm{m}}}{\tau_{0}}\right) \approx 23 \cdot \frac{k_{\mathrm{B}} T}{K}
$$

where $\tau_{0}$ is a time constant $\left(10^{-9} \mathrm{~s}\right), K$ the magnetic anisotropy constant (at $T=T_{\mathrm{B}}$ ) and $\tau_{\mathrm{m}}$ is the characteristic measurement time which, for SQUID magnetometer measurements, has an order of magnitude of $10 \mathrm{~s}$ (explaining the prefactor $23=10 \cdot \ln 10$ in the numerical application). Now, the contribution of blocked (BL) particles to the sample magnetization is [39]:

$$
M_{\mathrm{BL}}=\phi \frac{\mu_{0} \cdot M_{\mathrm{d}}^{2}}{3 K} H_{\mathrm{app}}
$$

where $\phi$ is the volume fraction of the sample. Then, the total magnetization of the sample $M(T)$ is given by the sum of $M_{\mathrm{BL}}$ and $M_{\mathrm{SPM}}[39]$ :

$$
\begin{gathered}
M_{\mathrm{BL}}=\int_{V_{B}}^{\infty} d r \cdot g_{\mathrm{d}_{0}, \sigma}(2 r) \cdot \phi \cdot \frac{\mu_{0} \cdot M_{\mathrm{d}}^{2}}{3 K} H_{\mathrm{app}} \\
M_{\mathrm{SPM}}=\int_{0}^{V_{B}} d r \cdot g_{\mathrm{d}_{0}, \sigma}(2 r) \cdot \phi \cdot \frac{\mu_{0} \cdot M_{\mathrm{d}}^{2} \cdot V_{\mathrm{M}}}{3 k_{\mathrm{B}} T} H_{\mathrm{app}} \\
M(T)=M_{\mathrm{BL}}+M_{\mathrm{SPM}}
\end{gathered}
$$

In a normal ZFC experiment, the temperature is varied from 5 to $300 \mathrm{~K}$ and hence, in equation (18), the domain magnetization $M_{\mathrm{d}}$ and anisotropy constant $K$ cannot be considered 
temperature independent. The domain magnetization is assumed to change with temperature according to a modified Bloch's law [40]:

$$
M_{\mathrm{d}}(T)=M_{\mathrm{d}, \mathrm{T}=0} \cdot\left(1-f_{\mathrm{e}} \cdot T^{2}\right)
$$

where, $f_{\mathrm{e}}$ is an empirical parameter. The exponent 2 in equation (19) arises from finite size effects. Then for NPs with uniaxial symmetry, the crystal anisotropy constant has to vary with temperature according to the following formula [40, 41]:

$$
K(T)=K_{\mathrm{T}=0} \cdot\left(1-f_{\mathrm{e}} \cdot T^{2}\right)^{3}
$$

In order to fit equation (19) to the experimental data, it is necessary to know the saturation magnetization $M_{\mathrm{sat}}(T=0)$ (or equivalently the nanoparticle domain magnetization $M_{\mathrm{d}, \mathrm{T}=0}$ which is just proportional) at zero temperature. Moreover, the phenomenological parameter $f_{\mathrm{e}}$ has to be known in order to calculate the temperature dependence of $S A R$. Therefore, the saturation magnetization (at 7 Tesla) was measured at different temperatures from 5 to 300 $\mathrm{K}$. These measurements were performed by the same commercial magnetometer (Quantum Designs MPMS-7T) as in ZFC. Figure 8a represents the so obtained $M_{\mathrm{d}}$ values obtained by dividing $M_{\text {sat }}$ by the volume fraction $\phi$ at different temperatures, properly fitted by equation (19). The $f_{\mathrm{e}}$ values that best fit experimental data to equation (19) are written in table 4.

(a)

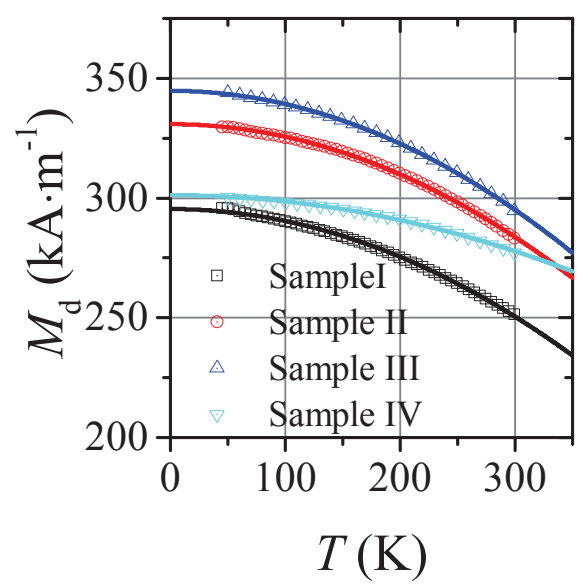

(b)

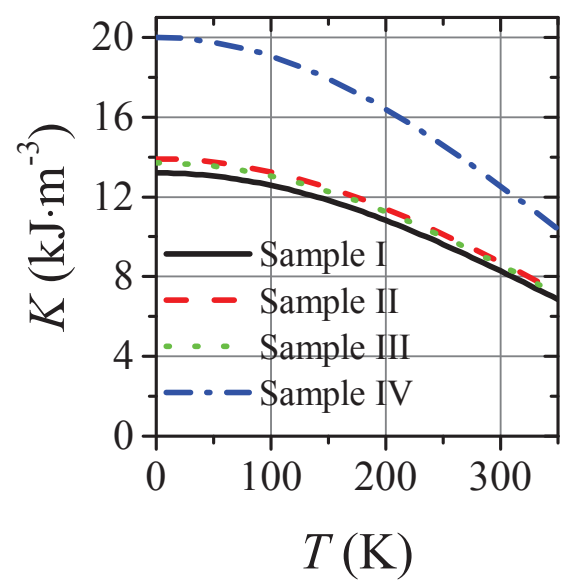

Figure 8: Measured $M_{d}$ at different sample temperatures. The curves are fitted with eq. (19) (see text); $b$ ) Temperature evolution of anisotropy constant $K$ according to eq. (20).

Figure 9 shows ZFC-FC curves for the four samples from 50 to $300 \mathrm{~K}$ measured by SQUID magnetometer. The samples were diluted 10 times to reduce inter-particle interactions. The ZFC branches were fitted by eq. (18), which is the sum of the magnetization $M_{\mathrm{BL}}$ of blocked moments (eq. 16) and $M_{\mathrm{SPM}}$ of unblocked moments (eq. 17). The two integrals on the size distribution have a lower (respectively upper) size limit (equivalently volume $V_{\mathrm{B}}$ ) taken as fit- 
Table 4: $f_{e}$ parameters that best fit to equation (19).

\begin{tabular}{cc}
\hline Sample & $f_{\mathrm{e}}\left(\mathrm{K}^{-2} \times 10^{-6}\right)$ \\
\hline I & $1.7 \pm 0.1$ \\
II & $1.6 \pm 0.1$ \\
III & $1.6 \pm 0.1$ \\
IV & $0.9 \pm 0.1$ \\
\hline
\end{tabular}

ting parameter, whereas the domain magnetization $M_{\mathrm{d}}(T)$ was fitted previously with eq. (19), of parameters $M_{\mathrm{d}, \mathrm{T}=0}$ and $f_{\mathrm{e}}$. By this method, the fitted volume $V_{\mathrm{B}}$ gave the anisotropy constant at $T=T_{\mathrm{B}}$ through eq. (14). The anisotropy constant at $T=0 \mathrm{~K}$ and at room temperature ( $T=300 \mathrm{~K}$ ) was obtained by eq. (20) with the $f_{\mathrm{e}}$ value already determined. It must be noted that the temperature dependence of $M_{\mathrm{d}}$ and of the anisotropy constant $K$ given respectively by eq. (19) and (20) are necessary to achieve a good agreement between experimental data and theory. Figure $8 \mathrm{~b}$ shows the variation of the obtained anisotropy constant with the temperature according to equation (20). Table 5 summarises the obtained $M_{\mathrm{d}}$ and anisotropy constant $K$ values at $T=0$ and at room temperature.

The anisotropy constants at room temperature respectively below (samples I, II, III) and above (sample IV) $10^{4} \mathrm{~J} \cdot \mathrm{m}^{-3}$ are compatible with values from literature on similar iron oxide nanoparticles measured by several methods such as SQUID magnetometry $[42,43]$ or ferromagnetic resonance [44].

Table 5: Measured anisotropy constant $K$ values deduced from curve fittings of the ZFC curve with eq. (18).

\begin{tabular}{ccc}
\hline & \multicolumn{2}{c}{$K\left(\mathrm{~kJ} \cdot \mathrm{m}^{-3}\right)$} \\
& $T=0 \mathrm{~K}$ & $T=300 \mathrm{~K}$ \\
\hline sample I & 13.2 & 8.3 \\
sample II & 13.9 & 8.8 \\
sample III & 13.7 & 8.6 \\
sample IV & 20.0 & 15.5 \\
\hline
\end{tabular}


(a) Sample I

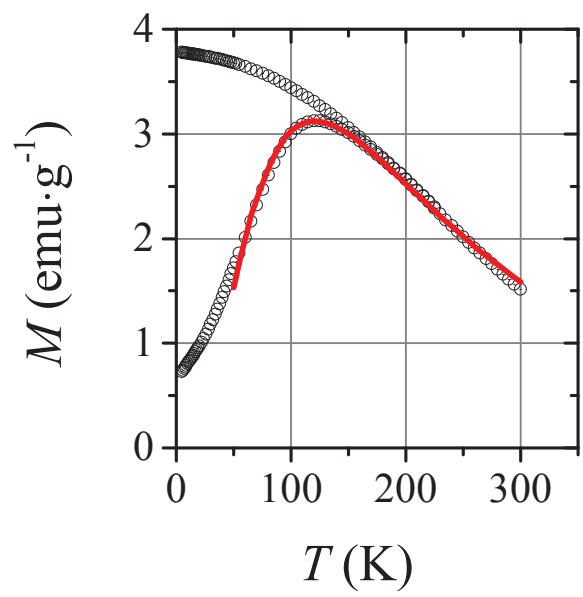

(b) Sample II

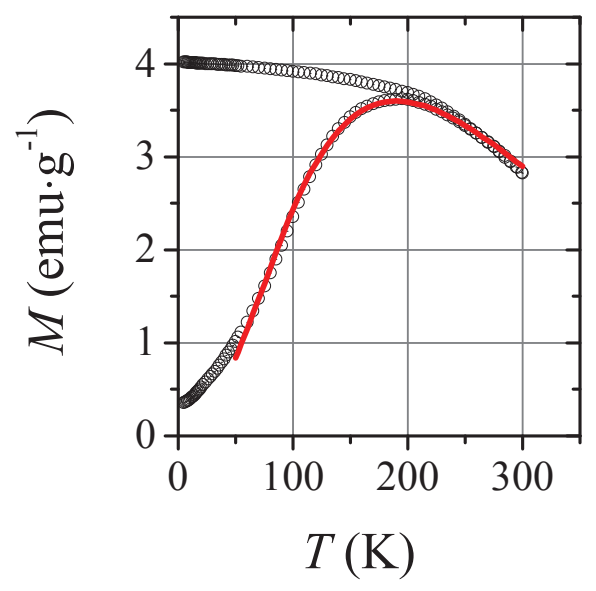

(d) Sample IV

(c) Sample III

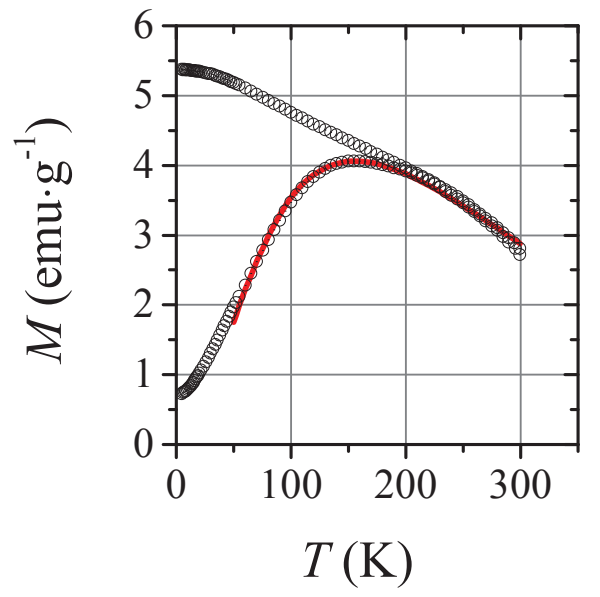

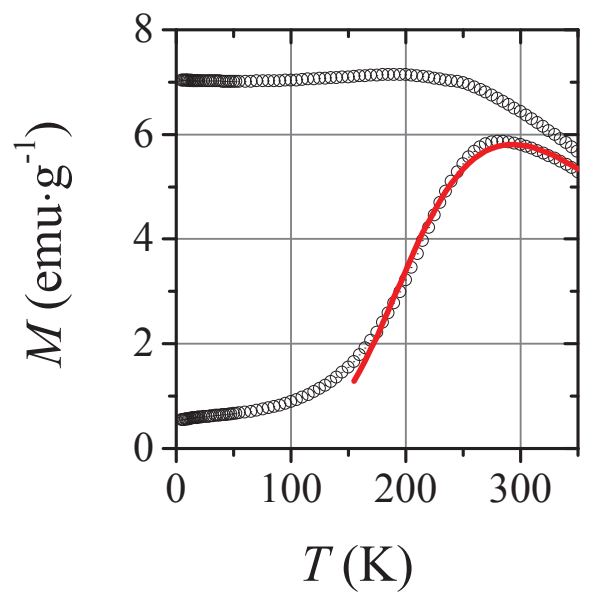

Figure 9: ZFC-FC curves of samples I, II, III and IV and their corresponding fitting of ZFC branch to equation (18) (solid lines). The magnetization was measured while applied field was $795.8 \mathrm{~A} \cdot \mathrm{m}^{-1}$ (10 Oe).

\subsection{Fitting of the experimentally measured Specific Absorption Rate}

The measured $S A R$ values were fitted to the non-interacting relaxation model previously described. The average size $d_{0}{ }^{\mathrm{VSM}}$ and $M_{\text {sat }}$ (or equivalently $M_{\mathrm{d}}$ ) values deduced from static $\mathrm{M}-\mathrm{H}$ curves (see section 3.2), were used as parameters determined independently. Also the anisotropy constant values previously obtained from the fits of the ZFC curves (see section 3.3) were used as fixed parameters. To obtain the theoretical thermal dependence of $S A R$, the temperature dependence of $M_{\mathrm{d}}$ and $K$ deduced in previous section (equations 19 and 20) were assumed with the corresponding $f_{\mathrm{e}}$ parameter. Regarding to hydrodynamic diameter, $d_{\mathrm{H}}$, the values obtained by DLS were used (see table 1). However, due to the short Néel relaxation time compared to the Brownian time constant calculated by eq. (4), the main relaxation mechanism is Néel relaxation process and the predicted $S A R$ value did not vary significantly with $d_{\mathrm{H}}$. It justifies the approximation made when considering the ferrofluid viscosity equal to the carrier fluid viscosity (water viscosity on table 2), in addition to the fact that volume fraction remains 
below $0.4 \%$ in all cases, therefore viscosity estimated by the Einstein relationship for solid suspension varies by less than $1 \%$. Finally, by means of the Levenberg-Marquardt non-linear fitting method, the standard size deviation $\sigma$ and precession time $\tau_{0}$ were varied to find out their respective values that best fit the experimental SAR data to the set of equations (4), (5), (6) and (7). Table 6 shows the so obtained standard size deviations $\sigma$ and $\tau_{0}$ values.

Table 6: Standard deviation of diameters $\sigma$ and characteristic $\tau_{0}$ values that best fit to the experimental SAR data with the combination of equations 4-20, in which all the other parameters were determined independently.

\begin{tabular}{ccc}
\hline & $\sigma(\mathrm{nm})$ & $\tau_{0}(\mathrm{~ns})$ \\
\hline sample I & 4.0 & 1.07 \\
sample II & 3.1 & 2.05 \\
sample III & 3.2 & 2.00 \\
sample IV & 3.4 & 0.43 \\
\hline
\end{tabular}

(a) Sample I

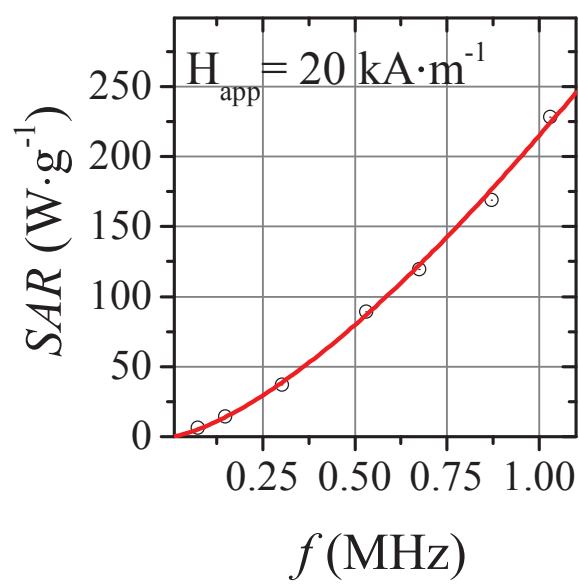

(c) Sample III

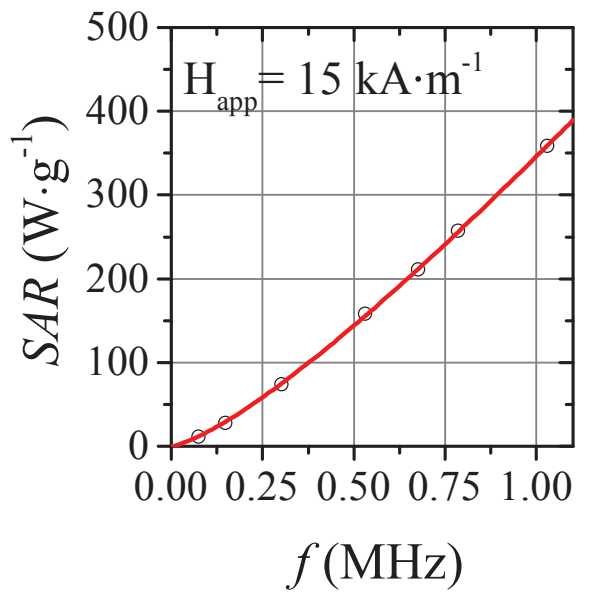

(b) Sample II

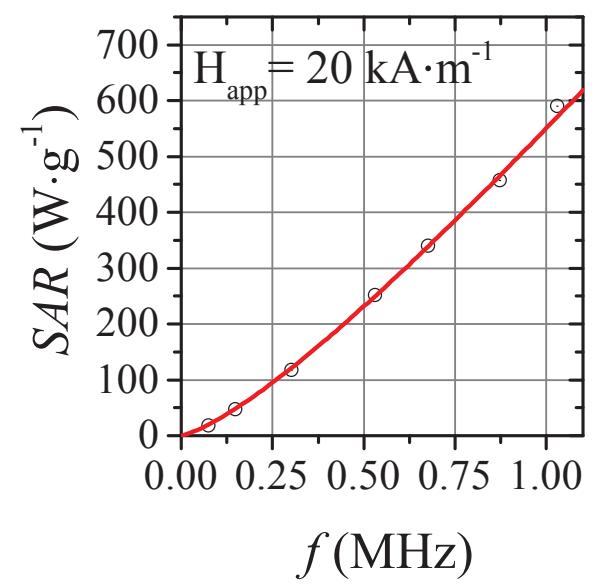

(d) Sample IV

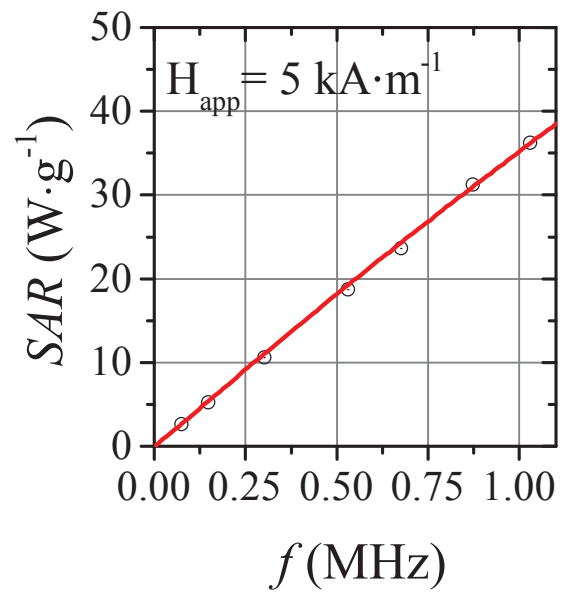

Figure 10: Measured SAR at a given field intensity versus frequency. Solid lines represent fitting with the curves predicted by the linear relaxation model using the magnetic parameters $d_{0}, K$ and $M_{d}$ measured independently. 
The standard deviation values in table 6 that best fit experimental data to equation (7) are different from the standard deviation values obtained from DC magnetization curves $\sigma^{\mathrm{VSM}}$; they are smaller, especially for sample IV. It was indeed impossible to properly adjust the experimental SAR curve with the $\sigma^{\mathrm{VSM}}$ values, which were too large.

Figure 10 represents measured SAR versus frequency curves for samples I, II, III and IV. This figure also shows the fitting of the experimental data using eq. (7). As it can be appreciated, the experimental data are in good agreement with the curves predicted by eq. (7) for all samples. On the other hand, graphs on figure 11 represent the measured thermal dependence $\triangle S A R_{\mathrm{T}}$ at 75,532 and $1030 \mathrm{kHz}$ as well as the fitting using equation (7) for each frequency. Experimental and theoretical data are in good agreement in the measured temperature range, from $10{ }^{\circ} \mathrm{C}$ to $60{ }^{\circ} \mathrm{C}$.

(a) Sample I

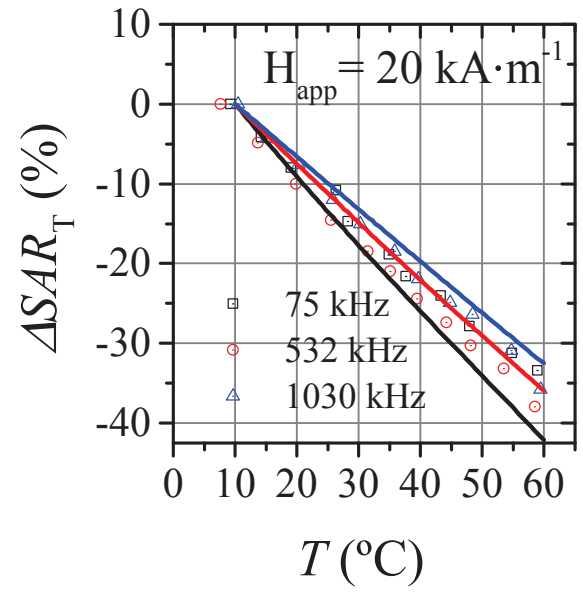

(c) Sample III

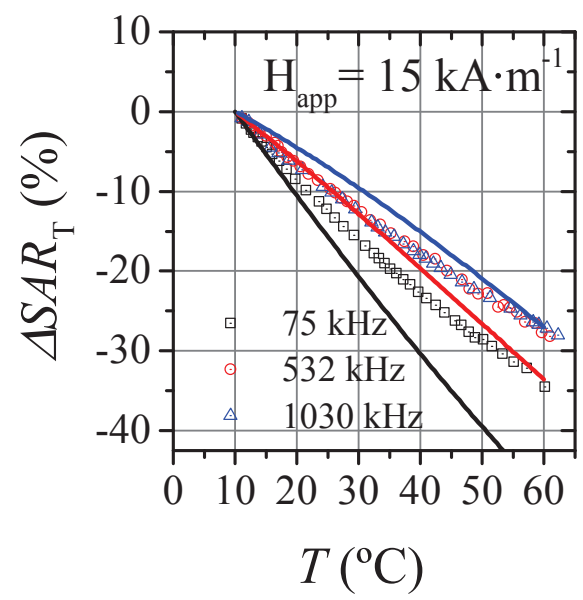

(b) Sample II

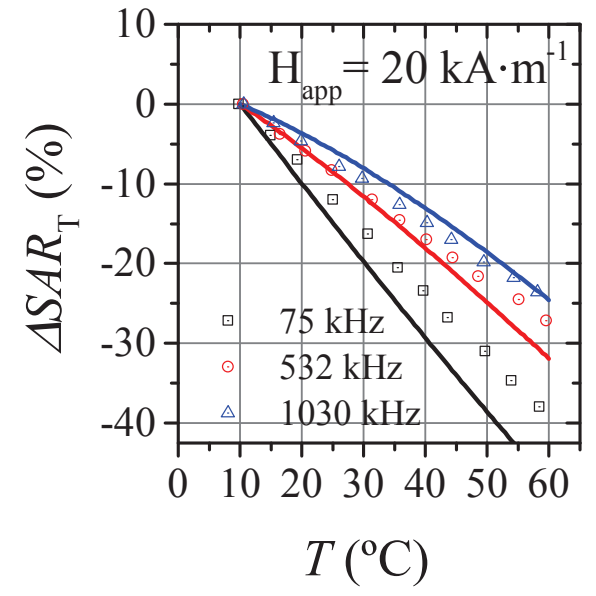

(d) Sample IV

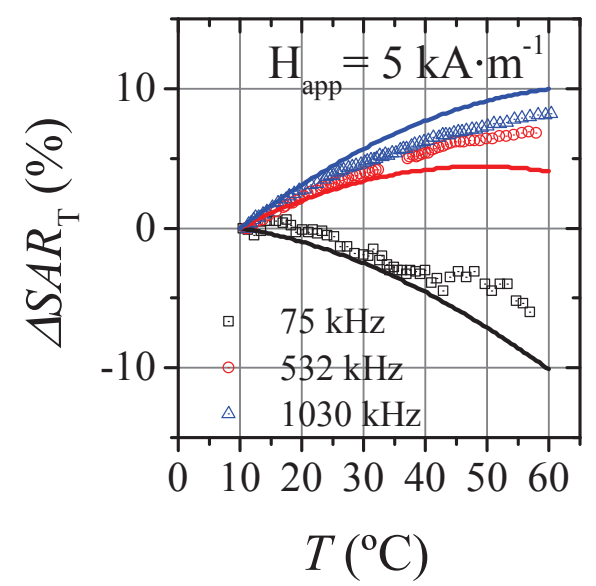

Figure 11: Measured $\triangle S A R_{T}$ at a given field intensity versus temperature. Solid lines represent the curves predicted by the linear relaxation model using the magnetic parameters $d_{0}, K$ and $M_{d}$ measured independently. 
To verify the linear relaxation model and especially to check the method to obtain the equilibrium $\chi_{0}$ from the static susceptibility of Langevin law, (see eq. 11), the SAR values measured at different applied magnetic field intensities where compared whit the values predicted by eq. (7). As it can be appreciated on figure 12, the experimental data are well fitted by the predicted values and hence, the method to calculate $\chi_{0}$ is adequate. In the case of sample IV dispersed in agarose gel, however, when magnetic field intensity rises above $6 \mathrm{kA} \cdot \mathrm{m}^{-1}$, the lineal response theory fails to predict the absorption rate.

(a) Sample I

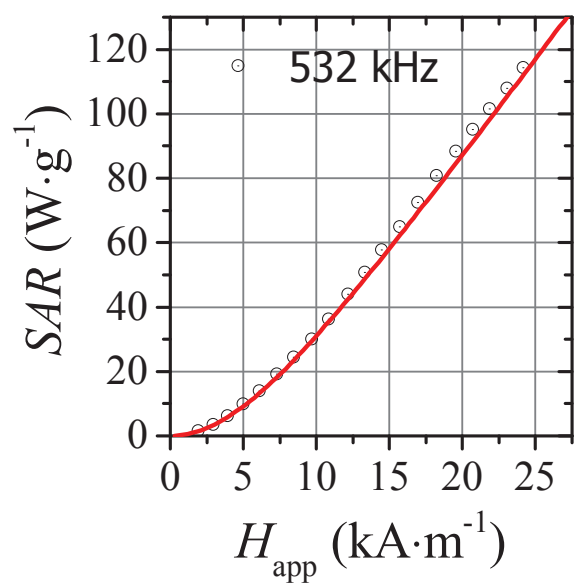

(c) Sample III

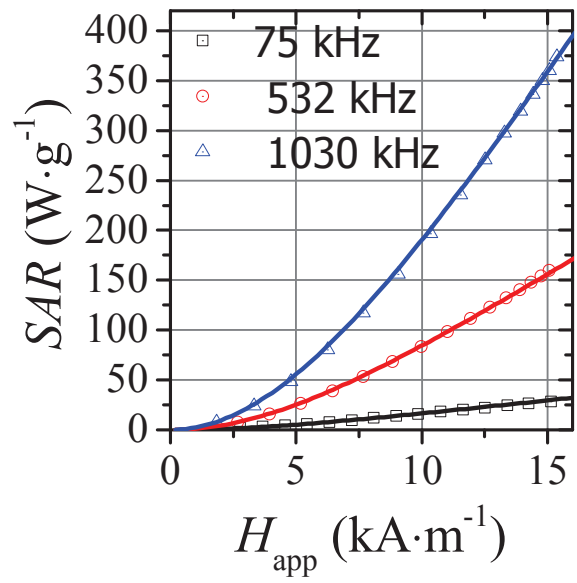

(b) Sample II

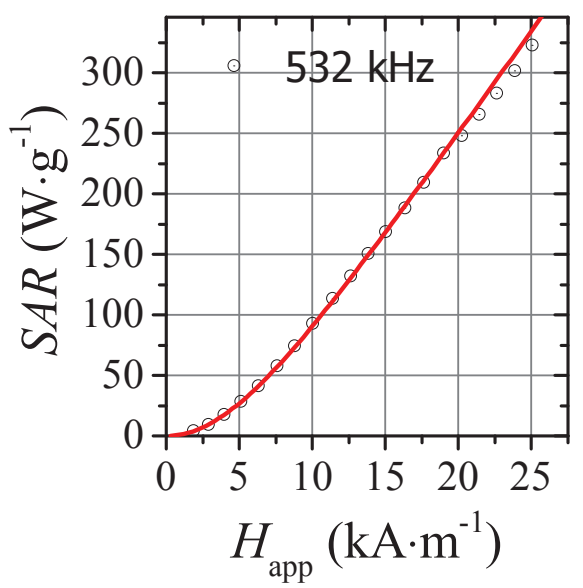

(d) Sample IV

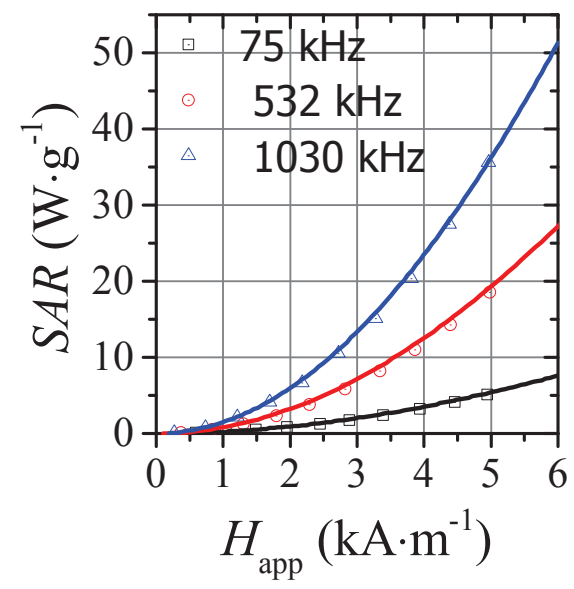

Figure 12: Measured SAR versus field intensity fitted by the predicted values (solid lines). Note that the lower SAR values in sample IV are due to lower $H_{\text {app }}$ values ( $x$-axis).

Finally, the variation of $S A R$ with temperature can be interpreted qualitatively from the theoretical increment with temperature of the optimum NP size, $d_{\mathrm{m}}$, which maximizes the specific loss power (see figure 13). The shifts are mainly ascribed to a change of relaxation times and, at a minor extend, to the decrease of the equilibrium susceptibility $\chi_{0}$ with a raise of temperature. According to eq. (7), SAR values must decrease with temperature when the experimental NP sizes are distributed below the optimal diameter $d_{\mathrm{m}}$. However, this situation reverses when the mean diameter becomes larger than $d_{\mathrm{m}}$, and thus, in that case, the thermal 
loss power should rather increase with temperature for a given frequency and field strength (see figure 13). The optimum diameter $d_{\mathrm{m}}$ also changes with the magnetic field frequency, explaining the reversal of the thermal behaviour for sample IV between $f=75 \mathrm{~Hz}$ and $f=1$ $\mathrm{MHz}$ (see the explanations in the legend of figure 13).

(a) $75 \mathrm{kHz}$

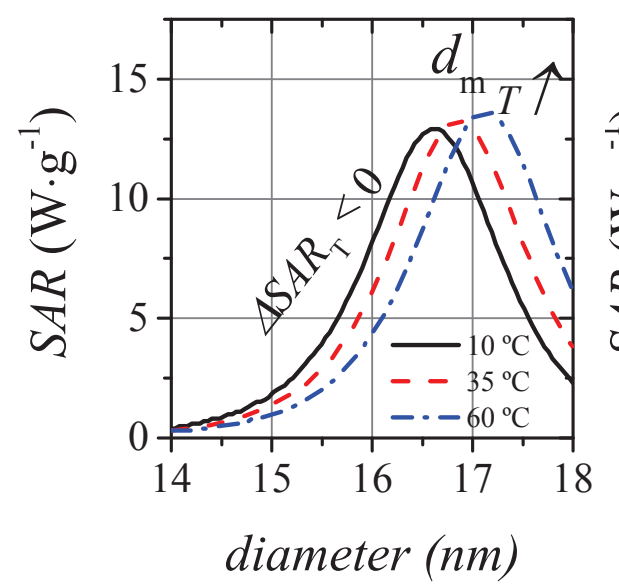

(b) $1030 \mathrm{kHz}$

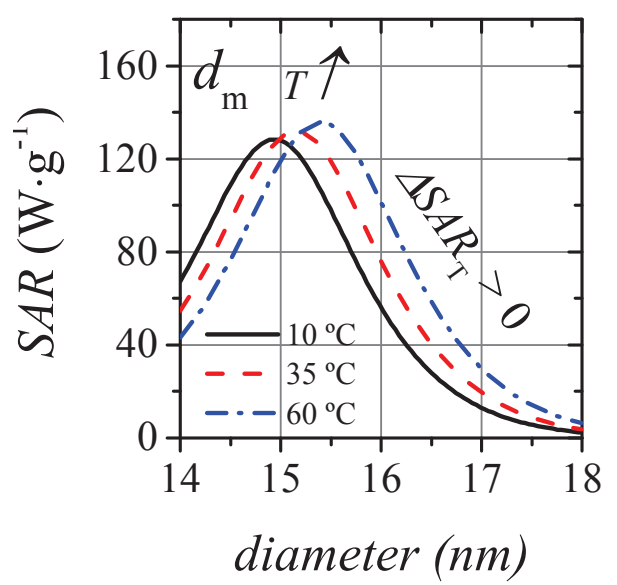

Figure 13: Predicted SAR values by eq. (7) versus monodisperse nanoparticle size for sample IV at a) $75 \mathrm{kHz}$ and b) $1 \mathrm{MHz}$. The magnetic field intensity is $H_{\text {app }}=5 \mathrm{kA} \cdot \mathrm{m}^{-1}$. At both frequencies, the optimal diameter $d_{m}$ shifts towards larger values when $T$ increases. For sample IV, the NPs are distributed in a range of sizes $16.1 \pm 3.4 \mathrm{~nm}$. These sizes are located mainly below $d_{m}$ at $f=75 \mathrm{kHz}$ and above $d_{m}$ at $f=1 \mathrm{MHz}$. Thus one expects a decrease of $S A R$ with $T$ at $f=75 \mathrm{kHz}$ and an increase of $S A R$ with $T$ at $f=1 \mathrm{MHz}$, as experimentally observed (figure 11).

\subsection{Out of linear theory}

In the case of sample IV, $\triangle S A R_{\mathrm{T}}$ depends on the applied magnetic field intensity $H_{\text {app }}$ as shown in figure 14, contrary to samples I, II and III. The linear response theory used to explain measured $S A R$ data in the previous section fails to explain this behaviour (see figure 14). Moreover, the measured $S A R$ values do not adjust to the theoretical curve predicted by the model when the field intensity $H_{\text {app }}$ exceeds $6 \mathrm{kA} \cdot \mathrm{m}^{-1}$ in sample IV. Therefore, a two-level model proposed by Carrey et al. [45] was used to explain the observed thermal behaviour. First, the dynamic time evolution of magnetization was calculated numerically for randomly oriented single domain nanoparticles. Then, when the simulated hysteresis loops converged [45], which happens after 5 periods, the $S A R$ was calculated by means of equation (2) for each temperature.

To carry out the numerical calculations, the values of anisotropy constant $K$, attempt of time $\tau_{0}$, domain magnetization $M_{\mathrm{d}}$ and size distribution parameters $\left(d_{0}, \sigma\right)$ obtained for sample IV in the previous sections were considered as parameters. Figure 15 shows the so obtained theoretical $\triangle S A R_{\mathrm{T}}$ values at $1 \mathrm{MHz}$ frequency. As it can be appreciated in figure 15 , there is a good qualitative agreement between experimental and simulated data, although a quantitative 
(a)

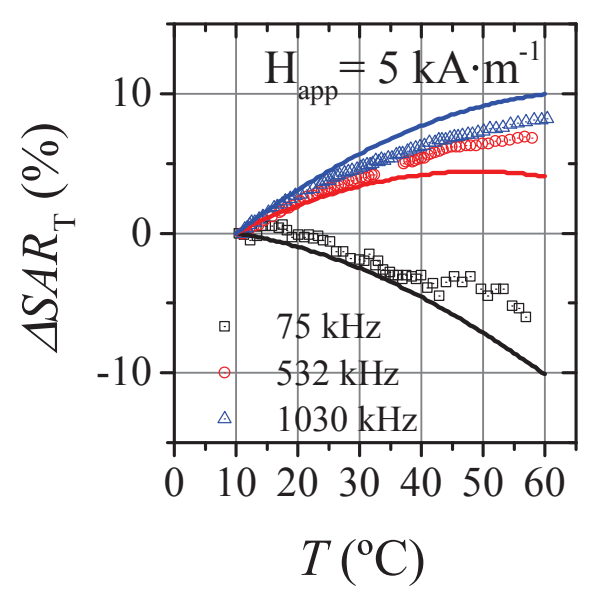

(b)

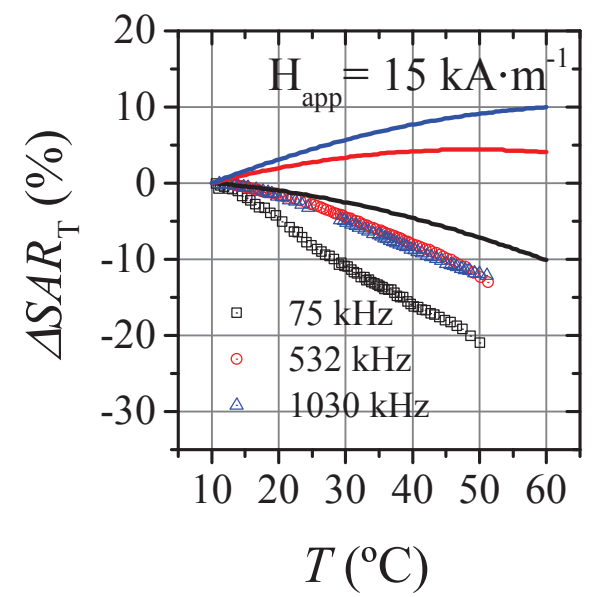

Figure 14: Measured $\triangle S A R_{T}$ of sample IV at a) $5 \mathrm{kA} \cdot \mathrm{m}^{-1}$ and $\left.\mathrm{b}\right) 15 \mathrm{kA} \cdot \mathrm{m}^{-1}$. Solid lines represent the theoretical curves predicted by the linear response model explained in the previous sections. For large field, the model fails to predict the experimental $\triangle S A R_{T}$.

discrepancy exists. One possible reason for the observed discrepancy is the assumption made in the two-level model: all the possible states are restricted to two discrete levels only [45, 46].

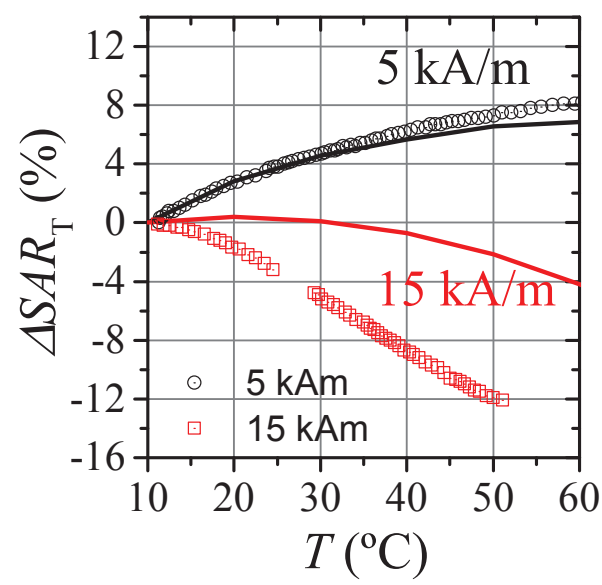

Figure 15: $\Delta S A R_{T}$ values predicted by the two-level model at $1030 \mathrm{kHz}$ frequency for sample IV (solid lines) and the corresponding experimental values.

The measured behaviour of $\Delta S A R_{\mathrm{T}}$ can be additionally interpreted as a reduction of $\Delta E$ barrier that separates two possible energy levels (1 and 2 in figure 16). Figure 16a shows the energy barrier $\Delta E$ when an external low magnetic field $\left(5 \mathrm{kA} \cdot \mathrm{m}^{-1}\right)$ is applied. Figure $16 \mathrm{~b}$, however, shows the energy barrier $\Delta E$ when an external stronger magnetic field is applied (15 $\left.\mathrm{kA} \cdot \mathrm{m}^{-1}\right)$. The barrier $\Delta E$ gets smaller when increasing the magnetic field intensity. On the other hand, the time that the magnetization needs to recover the equilibrium state, defined as Néel relaxation time, strongly depends on $\Delta E$. Indeed, the term $K V_{M}$ in equation (5) can be understood as the energy barrier $\Delta E[45,46]$ in figure 16 . Hence, Néel relaxation time $\tau_{\mathrm{N}}$ shortens when increasing the externally applied magnetic field intensity, resulting in a change 
of $\triangle S A R_{\mathrm{T}}$ behaviour with field intensity.

(a)

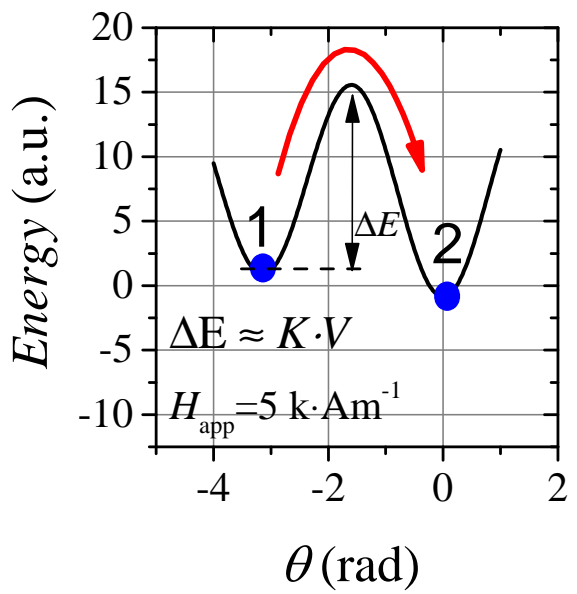

(b)

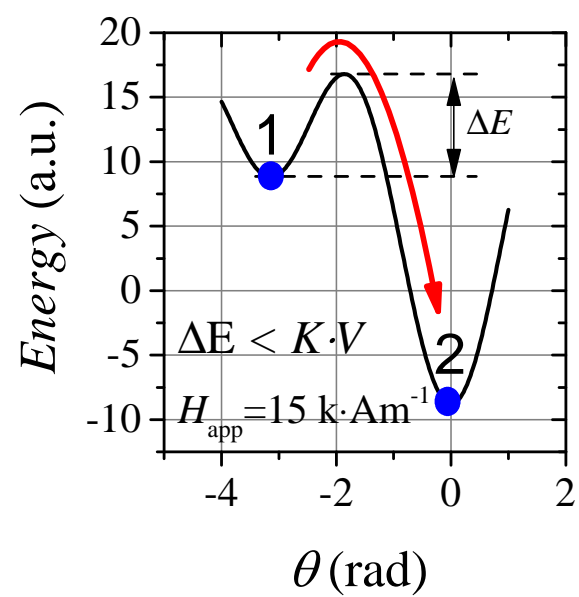

Figure 16: Energy as function of $\theta$, the angle between an easy magnetization-axis and the magnetic moment of the single domain nanoparticle with uniaxial anisotropy [45] $\left(K=10 \mathrm{~kJ} \cdot \mathrm{m}^{-3}\right)$. For simplicity the applied magnetic field is supposed parallel to easy magnetization-axis. The magnetic field intensity is a) $5 \mathrm{kA} \cdot \mathrm{m}^{-1}$ and b) $15 \mathrm{kA} \cdot \mathrm{m}^{-1}$.

The obtained results indicate clearly that $S A R$ values are dependent on temperature. The temperature dependence of $S A R$ implies some experimental impact on medical practice for tumour ablation. The heating efficiency of iron oxide nanoparticles can vary by up to $16 \%$ between $25{ }^{\circ} \mathrm{C}$ and $45{ }^{\circ} \mathrm{C}$, as reported in the present work. Therefore, in order to design more adequately magnetic hyperthermia treatments, the $S A R$ values measured at typical hyperthermia temperatures above the physiological temperature (from 41 to $46{ }^{\circ} \mathrm{C}$ ) have to be considered, rather than $S A R$ values measured at room temperature $\left(25^{\circ} \mathrm{C}\right)$. Although the difference is moderate, it can induce an error in the estimate final temperature of tissues under hyperthermia treatment, which can lead to undesired effects. On the other hand, the thermal dependence of $S A R$ could explain some discrepancies between values reported in the literature by different groups on analogous samples, especially when $S A R$ was obtained by calorimetric methods and assumed to be temperature independent. The present work aims at understanding the nature and causes of these $S A R$ variations with temperature in iron oxide nanoparticles, which are the most commonly used magnetic nanoparticles in view of their proven biocompatibility.

\section{Conclusions}

The specific heating efficiency ( $S A R$ value) of water-dispersed maghemite nanoparticles were measured at different temperatures and varying field strengths by AC magnetometry in a wide frequency range from 75 to $1030 \mathrm{kHz}$. From this study, we can conclude that it is possible to measure and to explain both qualitatively and quantitatively the temperature dependence of 
$S A R$ for superparamagnetic iron oxide (maghemite) nanoparticles.

It has been observed that the $S A R$ values of maghemite nanoparticles decrease with temperature when their sizes range mainly below $d_{\mathrm{m}}$, the optimum size that maximises $S A R$. This is the case of the smaller sizes samples I, II and III. However, when the nanoparticle sizes are distributed above $d_{\mathrm{m}}, S A R$ increases with temperature. This is the case of the largest size sample IV at low magnetic field $\left(5 \mathrm{kA} \cdot \mathrm{m}^{-1}\right)$ and high frequency (532 and $\left.1030 \mathrm{kHz}\right)$, but not at low frequency $(75 \mathrm{kHz})$. The linear response theory involving Néel relaxation and Brownian time constants was used to fully explain the measured $S A R$ values. To do so, independent magnetic measurements of nanoparticle average sizes, domain magnetization and anisotropy constants were made before fitting theoretical $S A R$ curves to experimental data. A good agreement between the linear relaxation model and measured data was confirmed in a tri-dimensional space (magnetic field frequency, intensity and sample temperature)

The linear model, however, fails to explain the thermal dependence of SAR at larger magnetic field $\left(H_{\text {app }}>6 \mathrm{kA} \cdot \mathrm{m}^{-1}\right)$ for sample IV. A recent paper [47] reported indeed that for large sized magnetic nanoparticles, such failure of the linear model is observed beyond a threshold magnetic field value. It was observed that the variation rate of $S A R$ with temperature changes of sign depending on the applied magnetic field intensity: it is positive at low field $\left(H_{\text {app }}=5\right.$ $\left.\mathrm{kA} \cdot \mathrm{m}^{-1}\right)$ whereas it is negative at lower field $\left(H_{\text {app }}=15 \mathrm{kA} \cdot \mathrm{m}^{-1}\right)$. At this point, a two-level energy model developed by J. Carrey et al. was used to explain the measured SAR dependence with temperature. A good qualitative and semi-quantitative agreement between experimental values and the values predicted by the model was achieved.

In conclusion, the presented AC magnetometer offers a practical method to directly measure $S A R$ values of magnetic nanoparticles at different temperatures and dispersed in various media, including water. This instrument can be particularly interesting to study subtle effects reported recently in the literature, such as the concentration-dependence of $S A R$, ascribed to magnetic dipolar interactions [48], or the effect of a thermally insulating materials wrapping the iron oxide cores, as evidenced with lipid bilayers [49]. The precise assessment of SAR is of utmost importance when preparing experiments of magnetic hyperthermia in vitro or in vivo, where the intrinsic properties of the utilized magnetic NPs need to be known beforehand in order to find out the necessary concentration of NPs and magnetic field conditions to deposit a given thermal dose. The obtained temperature dependence of $S A R$ values can induce an error in the final temperature estimation on tissues under hyperthermia treatment, which can lead to undesired effects. Moreover, the thermal dependence of SAR could explain some discrepancies between values reported in the literature by different groups on analogous samples, especially when $S A R$ was obtained by calorimetric methods and assumed to be temperature independent. Until now in the literature, the most precise parametrical studies of SAR values of SPM nanoparticles were two-dimensional (i.e. with only the field strength and frequency as coordinates) [50]. To our knowledge, this is the first tri-dimensional parametrical study (i.e., versus field strength, frequency and temperature), that shows total quantitative agreement between the experiments 
and the linear (Debye-type) model of magnetic moments relaxation.

\section{Acknowledgments}

We thank the Nanotex Company in Madrid for providing us with sample IV. We also want to acknowledge the Basque government for financial support under grant IT-443-10.

\section{References}

[1] R. Hergt, W. Andra, C.G. d'Ambly, I. Hilger, W.A. Kaiser, U. Richter, and H.-G. Schmidt. Physical limits of hyperthermia using magnetite fine particles. IEEE Transactions on Magnetics, 34(5):3745 - 3754, 1998.

[2] R E Rosenweig. Heating magnetic fluid with alternating magnetic field. Journal of Magnetism and Magnetic Materials, 252:370, 2002.

[3] Ming Ma, Ya Wu, Jie Zhou, Yongkang Sun, Yu Zhang, and Ning Gu. Size dependence of specific power absorption of fe3o4 particles in ac magnetic field. Journal of Magnetism and Magnetic Materials, 268(1-2):33 - 39, 2004.

[4] Jr. Lima, E., T.E. Torres, L.M. Rossi, H.R. Rechenberg, T.S. Berquo, A. Ibarra, C. Marquina, M.R. Ibarra, and G.F. Goya. Size dependence of the magnetic relaxation and specific power absorption in iron oxide nanoparticles. Journal of Nanoparticle Research, 15(5):1 - 11, 2013.

[5] Jean-Paul Fortin, Claire Wilhelm, Jacques Servais, Christine Menager, Jean-Claude Bacri, and Florence Gazeau. Size-sorted anionic iron oxide nanomagnets as colloidal mediators for magnetic hyperthermia. Journal of the American Chemical Society, 129(9):2628 2635, 2007.

[6] Eiji Kita, Shinji Hashimoto, Takeru Kayano, Makoto Minagawa, Hideto Yanagihara, Mikio Kishimoto, Keiichi Yamada, Tatsuya Oda, Nobuhiro Ohkohchi, Toshiyuki Takagi, Toshiyuki Kanamori, Yoshio Ikehata, and Isamu Nagano. Heating characteristics of ferromagnetic iron oxide nanoparticles for magnetic hyperthermia. Journal of Applied Physics, 107(9):09B321, 2010.

[7] Shu-Yi Wang, Shujuan Huang, and Diana-Andra Borca-Tasciuc. Potential sources of errors in measuring and evaluating the specific loss power of magnetic nanoparticles in an alternating magnetic field. IEEE Transactions on magnetics, 49(1, Part 2):255 - 262, 2013. 
[8] Eva Natividad, Miguel Castro, and Arturo Mediano. Adiabatic magnetothermia makes possible the study of the temperature dependence of the heat dissipated by magnetic nanoparticles under alternating magnetic fields. Applied Physics Leters, 98(24):243119, 2011.

[9] Eva Natividad, Miguel Castro, Graziella Goglio, Irene Andreu, Romain Epherre, Etienne Duguet, and Arturo Mediano. New insights into the heating mechanisms and selfregulating abilities of manganite perovskite nanoparticles suitable for magnetic fluid hyperthermia. Nanoscale, 4:3954 - 3962, 2012.

[10] R. Regmi, A. Naik, J. S. Thakur, P. P. Vaishnava, and G. Lawes. Temperature dependent dissipation in magnetic nanoparticles. Journal of Applied Physics, 115(17):17B301, 2014.

[11] B. Mehdaoui, J. Carrey, M. Stadler, A. Cornejo, C. Nayral, F. Delpech, B. Chaudret, and M. Respaud. Influence of a transverse static magnetic field on the magnetic hyperthermia properties and high-frequency hysteresis loops of ferromagnetic feco nanoparticles. Applied Physics Letters, 100(5):052403, 2012.

[12] S. A. Gudoshnikov, B. Ya Liubimov, and N. A. Usov. Hysteresis losses in a dense superparamagnetic nanoparticle assembly. AIP advances, 2(1):012143, 2012.

[13] S. A. Gudoshnikov, B. Y. Liubimov, Y. S. Sitnov, V. S. Skomarovsky, and N. A. Usov. Ac magnetic technique to measure specific absorption rate of magnetic nanoparticles. Journal of Superconductivity and Novel Magnetism, 26(4, SI):857 - 860, 2013.

[14] M. Veverka, P. Veverka, O. Kaman, A. Lancok, K. Zaveta, E. Pollert, K. Knizek, J. Bohacek, M. Benes, P. Kaspar, E. Duguet, and S. Vasseur. Magnetic heating by cobalt ferrite nanoparticles. Nanotechnology, 18(34):345704, 2007.

[15] Milolos Bekovik, Mladen Trlep, Marko Jesenik, Viktor Gorican, and Anton Hamler. An experimental study of magnetic-field and temperature dependence on magnetic fluid's heating power. Journal of Magnetism and Magnetic Materials, 331(0):264 - 268, 2013.

[16] E. Garaio, J.M. Collantes, J.A. Garcia, F. Plazaola, S. Mornet, F. Couillaud, and O. Sandre. A wide-frequency range ac magnetometer to measure the specific absorption rate in nanoparticles for magnetic hyperthermia. Journal of Magnetism and Magnetic Materials, 2013.

[17] R Massart. Preparation of aqueous magnetic liquids in alkaline and acid media. IEEE Transactions on Magnetics, 17(2):1247 - 1248, 1981.

[18] F Tourinho, R Franck, R Massart, and R Prezynski. Synthesis and magnetic-properties of manganese and cobalt ferrite ferrofluids. Progress in Colloid and Polymer Science", 79:128 - 134, 1989. 
[19] E Dubois, V Cabuil, F Boue, and R Perzynski. Structural analogy between aqueous and oily magnetic fluids. Journal of Chemical Physics, 111(15):7147 - 7160, 1999.

[20] R Massart, E Dubois, V Cabuil, and E Hasmonay. reparation and properties of monodisperse magnetic fluids. Journal of Magnetism and Magnetic Materials, 149:1 - 5, 1995.

[21] Paolo Arosio, Julie Thevenot, Tomas Orlando, Francesco Orsini, Maurizio Corti, Manuel Mariani, Lorenzo Bordonali, Claudia Innocenti, Claudio Sangregorio, Hugo Oliveira, Sebastien Lecommandoux, Alessandro Lascialfari, and Olivier Sandre. Hybrid iron oxidecopolymer micelles and vesicles as contrast agents for mri: impact of the nanostructure on the relaxometric properties. J. Mater. Chem. B, 1:5317 - 5328, 2013.

[22] QL Vuong, JF Berret, J Fresnais, Y Gossuin, and O Sandre. A universal scaling law to predict the efficiency of magnetic nanoparticles as mri t2-contrast agents. Advanced Healthcare Materials, 1(4):502 - 512, 2012.

[23] M. Safi, H. Sarrouj, O. Sandre, N. Mignet, and J-F Berret. Interactions between sub-10$\mathrm{nm}$ iron and cerium oxide nanoparticles and $3 \mathrm{t} 3$ fibroblasts: the role of the coating and aggregation state. Nanotechnology, 21(14):145103(10pp), 2010.

[24] Robert S. Molday US patent 4,452,773 (1984) "Magnetic iron-Dextran microspheres".

[25] S Mornet, J Portier, and E Duguet. A method for synthesis and functionalization of ultrasmall superparamagnetic covalent carriers based on maghemite and dextran. Journal of Magnetism and Magnetic Materials, 293(1, SI):127 - 134, 2005.

[26] F. Plazaola, E. Garaio, J. M. Collantes, I. Castellanos, M. Insausti, I. Gil de Muro, and J. A. Garcia. Specific absorption rate of magnetite nanoparticle powders with and without surrounding organic ligands. Jouurnal of Nanoscience and Nanotehnology, 12(9):7451 $7455,2012$.

[27] E. Garaio, J.M. Collantes, F. Plazaola, J.A. Garcia, and I. Castellanos-Rubio. A multifrequency eletromagnetic applicator with an integrated $\mathrm{AC}$ magnetometer for magnetic hyperthermia experiments. Measurement Science and Technology, 25:115702 (10 pp.), 2014.

[28] Cecilia N. Obeada and I. Malaescu. The temperature effect on the combined brownian and neel relaxation processes in a water-based magnetic fluid. Physica B-condensed Matter, 424:69 - 72, 2013.

[29] JL Dorman. Le phénomène de superparamagnétisme. Revue de Physique Appliquee, 16(6):275 - 301, 1981.

[30] E.P. Wohlfarth. Magnetic properties of single domain ferromagnetic particles. Journal of Magnetism and Magnetic Materials, 39(1 - 2):39 - 44, 1983. 
[31] W.M. Haynes. CRC Handbook of Chemistry and Physics, 93rd Edition. CRC Handbook of Chemistry and Physics. Taylor \& Francis, 2012.

[32] R.W. Chantrell, J. Popplewell, and S.W. Charles. Measurements of particle size distribution parameters in ferrofluids. IEEE Transactions on Magnetics, 14(5):975 - 977, 1978.

[33] D. X. Chen, A. Sanchez, E. Taboada, A. Roig, N. Sun, and H. C. Gu. Size determination of superparamagnetic nanoparticles from magnetization curve. Journal of Applied Physics, 105(8):083924, 2009.

[34] K Haneda and AH Morrish. Magnetic-structure at gamma-fe2o3-organic interfaces. IEEE transactions on Magnetics, 16(1):50 - 52, 1980.

[35] JC Denardin, AL Brandl, $M$ Knobel, P Panissod, AB Pakhomov, H Liu, and XX Zhang. Thermoremanence and zero-field-cooled/field-cooled magnetization study of $\mathrm{Co}_{\mathrm{x}}\left(\mathrm{SiO}_{2}\right)_{1-\mathrm{x}}$ granular films. Physical Review B, 65(6), 2002.

[36] K. Nadeem, H. Krenn, T. Traussnig, R. Wuerschum, D. V. Szabo, and I. Letofsky-Papst. Spin-glass freezing of maghemite nanoparticles prepared by microwave plasma synthesis. Journal of Applied Physics, 111(11), JUN 12012.

[37] J. Clarke and A.I. Braginski. The SQUID Handbook: Fundamentals and Technology of SQUIDs and SQUID Systems. Number v. 1. Wiley, 2006.

[38] R. Cabassi, F. Bolzoni, and F. Casoli. Differential method for sample holder background subtraction in superconducting quantum interference device (SQUID) magnetometry. Meassurement Science and Technology, 21(3), 2010.

[39] M. Knobel, W. C. Nunes, L. M. Socolovsky, E. De Biasi, J. M. Vargas, and J. C. Denardin. Superparamagnetism and other magnetic features in granular materials: A review on ideal and real systems. Journal of Nanoscience and Nanotechnology, 8(6):2836 - 2857, 2008.

[40] Jun Wang, Hongyan Duan, Xiong Lin, Victor Aguilar, Aaron Mosqueda, and Guo-meng Zhao. Temperature dependence of magnetic anisotropy constant in iron chalcogenide fe3se4: Excellent agreement with theories. Journal of Applied Physics, 112(10):103905, 2012.

[41] Soshin Chikazumi. Physics of Ferromagnetism. Clarendon pres. Oxfor, 1997.

[42] Lenaic Lartigue, Claudia Innocenti, Thangavel Kalaivani, Azzam Awwad, Maria del Mar Sanchez Duque, Yannick Guari, Joulia Larionova, Christian Guérin, Jean-Louis Georges Montero, Véronique Barragan-Montero, Paolo Arosio, Alessandro Lascialfari, Dante Gatteschi, and Claudio Sangregorio. Water-dispersible sugar-coated iron oxide nanoparticles: An evaluation of their relaxometric and magnetic hyperthermia properties. Journal of the American Chemical Society, 133(27):10459 - 10472, 2011. 
[43] A. Demortiere, P. Panissod, B. P. Pichon, G. Pourroy, D. Guillon, B. Donnio, and S. Begin-Colin. Size-dependent properties of magnetic iron oxide nanocrystals. Nanoscale, 3:225 - 232, 2011.

[44] F Gazeau, J.C Bacri, F Gendron, R Perzynski, Yu.L Raikher, V.I Stepanov, and E Dubois. Magnetic resonance of ferrite nanoparticles: evidence of surface effects. Journal of Magnetism and Magnetic Materials, 186(1 - 2):175 - 187, 1998.

[45] J. Carrey, B. Mehdaoui, and M. Respaud. Simple models for dynamic hysteresis loop calculations of magnetic single-domain nanoparticles: Application to magnetic hyperthermia optimization. Journal of Applied Physics, 110(3):083921, 2011.

[46] Yu. L. Raikher and V. I. Stepanov. Dynamic hysteresis of a superparamagnetic nanoparticle at low-to-intermediate frequencies. Journal of Magnetism and Magnetic Materials, 300(1):E311 - E314, 2006.

[47] Rudolf Hergt, Silvio Dutz, and Matthias Zeisberger. Validity limits of the Neel relaxation model of magnetic nanoparticles for hyperthermia. Nanotechnology, 21(1):015706, 2010.

[48] Yolanda Pineiro-Redondo, Manuel Banobre-Lopez, Ivan Pardinas-Blanco, Gerardo Goya, M. Arturo Lopez-Quintela, and Jose Rivas. The influence of colloidal parameters on the specific power absorption of PAA-coated magnetite nanoparticles. Nanoscale Research letters, 6:383, 2011.

[49] Gaelle Bealle, Riccardo Di Corato, Jelena Kolosnjaj-Tabi, Vincent Dupuis, Olivier Clement, Florence Gazeau, Claire Wilhelm, and Christine Ménager. Ultra magnetic liposomes for mr imaging, targeting, and hyperthermia. Langmuir, 28(32):11843 - 11851, 2012.

[50] R. Di Corato, A. Espinosa, L. Lartigue, M. Tharaud, S. Chat, T. Pellegrino, C. Ménager, F. Gazeau, and C. Wilhelm. Magnetic hyperthermia efficiency in the cellular environment for different nanoparticle designs. Biomaterials, 35:6400 - 6411, 2014. 